\title{
The Penn State - Toruń Centre for Astronomy Planet Search stars $^{\star}, \star \star$
}

\section{The sample of evolved stars}

\author{
A. Niedzielski ${ }^{1}$, B. Deka-Szymankiewicz ${ }^{1}$, M. Adamczyk ${ }^{1}$, M. Adamów ${ }^{2,1}$, G. Nowak ${ }^{3,4,1}$, and A. Wolszczan ${ }^{5,6}$ \\ 1 Toruń Centre for Astronomy, Nicolaus Copernicus University in Toruń, Grudziadzka 5, 87-100 Toruń, Poland \\ e-mail: Andrzej.Niedzielski@umk.pl \\ 2 McDonald Observatory and Department of Astronomy, University of Texas at Austin, 2515 Speedway, Stop C1402, Austin, Texas, \\ 78712-1206, USA \\ 3 Instituto de Astrofísica de Canarias, C/ Vía Láctea, s/n, 38205 La Laguna,Tenerife, Spain \\ ${ }^{4}$ Departamento de Astrofísica, Universidad de La Laguna, 38206 La Laguna, Tenerife, Spain \\ 5 Department of Astronomy and Astrophysics, Pennsylvania State University, 525 Davey Laboratory, University Park, PA 16802, \\ USA \\ ${ }^{6}$ Center for Exoplanets and Habitable Worlds, Pennsylvania State University, 525 Davey Laboratory, University Park, PA 16802, \\ USA
}

Received 14 September 2015 / Accepted 19 October 2015

\begin{abstract}
Aims. We present the complete spectroscopic analysis of 455 stars observed within the Penn State - Torun Centre for Astronomy Planet Search (PTPS) with the High Resolution Spectrograph of the $9.2 \mathrm{~m}$ Hobby-Eberly Telescope. We also present the total sample of 744 evolved stars of the PTPS and discuss masses of stellar hosts in our and other surveys devoted to evolved planetary systems. Methods. Stellar atmospheric parameters were determined through a strictly spectroscopic LTE analysis of equivalent widths of Fe I and Fe II lines. Rotational velocities were obtained from fitting synthetic spectra. Radial velocities were obtained from fitting a Gaussian function to the cross-correlation function. We determined stellar masses, ages, and luminosities with a Bayesian analysis of theoretical isochrones. The radii were calculated either from derived masses and $\log g$ or from $T_{\text {eff }}$ and luminosities.

Results. We present basic atmospheric parameters $\left(T_{\text {eff }}, \log g, v_{\mathrm{t}}\right.$ and $\left.[\mathrm{Fe} / \mathrm{H}]\right)$, rotation velocities, and absolute radial velocities as well as luminosities, masses, ages and radii for 402 stars (including 11 single-line spectroscopic binaries) that are mostly subgiants and giants. For 272 of them we present parameters for the first time. For another 53 stars we present estimates of $T_{\text {eff }}$ and $\log g$ based on photometric calibrations. More than half of the objects were found to be subgiants, but there is also a large group of giants, and a few stars appear to be dwarfs. The results show that the sample is composed of stars with masses ranging from 0.52 to $3.21 M_{\odot}$, 17 of which have masses $\geq 2.0 M_{\odot}$. The stellar radii range from 0.66 to $36.04 R_{\odot}$, with the vast majority having radii between 2.0 and $4.0 R_{\odot}$. They are generally less metal abundant than the Sun with a median $[\mathrm{Fe} / \mathrm{H}]=-0.07$. For 62 stars that we have in common with other planet searches, the stellar atmospheric parameters we found agree very well. We also present basic properties of the complete list of 744 stars that form the PTPS sample of evolved stars. We examined stellar masses for 1255 stars in five other planet searches and found that some of them are probably significantly overestimated. From applying our uniformly determined stellar masses, we confirm the apparent increase of companion masses for evolved stars, and we explain this as well as the lack of close-in planets with the limited effective radial velocity precision for these stars that is due to their activity.
\end{abstract}

Key words. stars: atmospheres - stars: fundamental parameters - stars: late-type - stars: general

\section{Introduction}

After over 20 years of research, with 2000 planets found around other stars since the discoveries of the first extrasolar systems by Wolszczan \& Frail (1992), Mayor \& Queloz (1995), and Marcy \& Butler (1996), it appears clear that of all available

* Based on observations obtained with the Hobby-Eberly Telescope, which is a joint project of the University of Texas at Austin, the Pennsylvania State University, Stanford University, LudwigMaximilians-Universität München, and Georg-August-Universität Göttingen.

$\star \star$ Tables $1-5$ are only available at the CDS via anonymous ftp to cdsarc.u-strasbg.fr (130.79.128.5) or via

http://cdsarc.u-strasbg.fr/viz-bin/qcat?J/A+A/585/A73 observational techniques applied to searches for exoplanets, the precise radial velocity (RV) and stellar transits delivered most of data.

The RV technique has been proved to be especially useful in searching for planets around massive and evolved stars. Massive and intermediate-mass main-sequence (MS) stars have high effective temperatures and rotate rapidly. As a result of the paucity of spectral lines and their width, these stars are not suitable for high-precision RV searches for planetary companions. Unfortunately, planetary candidates around such stars, even if discovered occasionally in transit searches (cf. Borucki et al. 2011; Schwamb et al. 2013), are very difficult to confirm with RV measurements. Consequently, transit projects have delivered very few planetary systems around stars much more massive 
than the Sun, with Kepler-432 (Ciceri et al. 2015; Ortiz et al. 2015; Quinn et al. 2015), Kepler-435 (Almenara et al. 2015) being the most prominent examples so far.

Radial velocity searches that focus on giant and subgiant stars that evolve off the MS, cool down, and considerably slow their rotation, exhibit an abundant narrow-line spectrum that makes them accessible to RV techniques, which deliver most of data on such planetary systems. The slowly growing population of the currently known $\sim 60$ planets around evolved stars is a result of intense research in projects such as the McDonald Observatory Planet Search (Cochran \& Hatzes 1993; Hatzes \& Cochran 1993), the Okayama Planet Search (Sato et al. 2003), the Tautenberg Planet Search (Hatzes et al. 2005), the Lick K-giant Survey (Frink et al. 2002), the ESO FEROS planet search (Setiawan et al. 2003a,b), the Retired A Stars and Their Companions (Johnson et al. 2007), the Coralie \& HARPS search (Lovis \& Mayor 2007), the Boyunsen Planet Search (Lee et al. 2011), our own Pennsylvania-Toruń Planet Search (PTPS, Niedzielski et al. 2007; Niedzielski \& Wolszczan 2008), and several others.

The most massive hosts of planetary systems have almost exclusively been discovered by such surveys (e.g., Sato et al. 2007, 2010, 2012, 2013). These surveys have demonstrated, for example, a paucity of planets within $0.5 \mathrm{AU}$ of their parent stars (Johnson et al. 2007; Sato et al. 2008; Jones et al. 2014), a borderline currently broken by Kepler 91 b (Lillo-Box et al. 2014; Barclay et al. 2015). They also proved capable of delivering evidence for recent violent star-planet interactions in aging planetary systems (Adamów et al. 2012). We note, however, a slowly growing statistics of planetary systems around MS stars more massive than the Sun discovered in transit surveys (KELT-7 Bieryla et al. 2015; WASP-78 - Smalley et al. 2012; HET-P-40 - Hartman et al. 2012).

Stellar masses are essential to determine the minimum masses of planetary-mass companions $\left(m_{\mathrm{P}} \sin i\right)$ in all RV planet searches. Precise determinations of masses of isolated single MS stars are a difficult in themselves, but in the case of evolved stars, like subgiants or giants, they are even more difficult. Of all indirect methods for estimating stellar mass, asteroseismology is probably the most reliable. Unfortunately, the vast majoritys of stars that were searched for planets with the RV technique are not intensively enough studied for oscillations to deliver masses. This situation will hopefully improve with the Transiting Exoplanet Survey Satellite (TESS; Ricker et al. 2014) or PLATO 2.0 (Rauer et al. 2014), especially with the new, more precise parallaxes from Gaia (Gilmore et al. 1998; Perryman et al. 2001).

It is not surprising therefore that masses of evolved stars studied in planet searches are very uncertain. If systematic effects are present in addition to large uncertainties in stellar mass estimates, the problem may be even more severe as masses for some types of stars may be systematically incorrect. In this context it is important to note that one of the most striking features of known planetary systems around evolved stars is a significant stellar and companion-mass increase for more evolved stars (Niedzielski et al. 2015b). An average dwarf ${ }^{1}$ with a planetary system usually is a solar-mass F or G spectral type star. An average subgiant known to possess a planetary system is already a star with a mass of $\approx 1.5 M_{\odot}(\mathrm{MS}$ spectral type A-F), and an

\footnotetext{
After Niedzielski et al. (2015b), we call for simplicity stars with $\log g=4.5 \pm 0.5$ dwarf stars, those with $\log g=3.5 \pm 0.5$ subgiants, stars with $\log g=2.5 \pm 0.5$ giants, and those with $\log g=$ $1.5 \pm 0.5$ bright giants.
}

average giant with planets (MS A-type star) is almost twice as massive as a dwarf (5-10 $\sigma$ difference).

On the other hand, among the known stars with planets, an average giant hosts a companion about twice as massive as a dwarf, and a bright giant's companion is three times more massive on average. This places companions to bright giants, on average, at the brown dwarf - planet borderline (see also Mitchell et al. 2013). It is rather obvious that such an increase in giant planet mass cannot be explained by accretion during the red giant branch evolution of its host (Duncan \& Lissauer 1998).

The stellar mass is not expected to increase during the MS and the subgiant branch evolution. Therefore stellar masses of evolved stars in planet searches are sometimes considered to be overestimated (Lloyd 2011, 2013; Schlaufman \& Winn 2013). This is a very important point as uncertainties in stellar masses immediately lead to uncertainties in planetary masses and in consequence make statistical considerations of exoplanets more difficult. Sousa et al. (2015) recently considered masses of hosts of known planetary systems in more detail and found some of them to be overestimated. Here we present a more general approach to this problem.

With this paper we continue a series dedicated to the detailed description of the complete sample of $\sim 1000$ stars studies within PTPS. This project is performed with the Hobby-Eberly Telescope (HET, Ramsey et al. 1998) and is devoted to planets in evolved planetary systems. So far, 20 planets in 17 systems have been found (Niedzielski et al. 2007, 2009a,b; Gettel et al. 2012b,a; Adamów et al. 2012; Nowak et al. 2013; Niedzielski et al. 2015a,b).

In the first paper of this series we presented the red giant clump (RGC) sample (Zieliński et al. 2012 - hereafter Paper I). In the second one we presented $\mathrm{Li}$ abundances in that sample (Adamów et al. 2014 - Paper II). The purpose of the present paper is to deliver physical parameters, such as effective temperatures $\left(T_{\text {eff }}\right)$, stellar gravitational accelerations $(\log g)$, microturbulence velocities $\left(v_{\mathrm{t}}\right)$, and metallicities $([\mathrm{Fe} / \mathrm{H}])$ for $455 \mathrm{GK}$ type stars that presumably are subgiants and red giants observed within PTPS survey. The atmospheric parameters, together with existing photometric data and parallaxes (when available) allow us to estimate stellar masses $\left(M / M_{\odot}\right)$, radii $\left(R / R_{\odot}\right)$, and ages. We also discuss the complete PTPS sample of evolved stars and stellar masses in this and several other planet searches.

The paper is structured as follows: in Sect. 2 we describe the sample and the observational material we used in the analysis. The spectroscopic analysis of the collected data is described in more detail in Sect. 3. In Sect. 4 we present the stellar integrated parameters: masses, luminosities, ages, and radii. Section 5 contains a short description of the complete PTPS sample of evolved stars, while in Sect. 6 we compare the sample to other samples of evolved stars that were searched for planets, for which basic data are available in the literature. In Sect. 7 we discuss our results and analyze the origin of planetary mass increases apparent in evolved planetary systems in more detail. Section 8 contains short conclusions.

\section{Targets selection and observations}

The spectroscopic observations presented here were made with the HET and its High Resolution Spectrograph (HRS, Tull 1998) in the queue-scheduled mode (Shetrone et al. 2007). The spectrograph was used in the $R=60000$ resolution mode and was fed with a 2 arcsec fiber. The configuration and observing procedure were identical to those described in Paper I. 

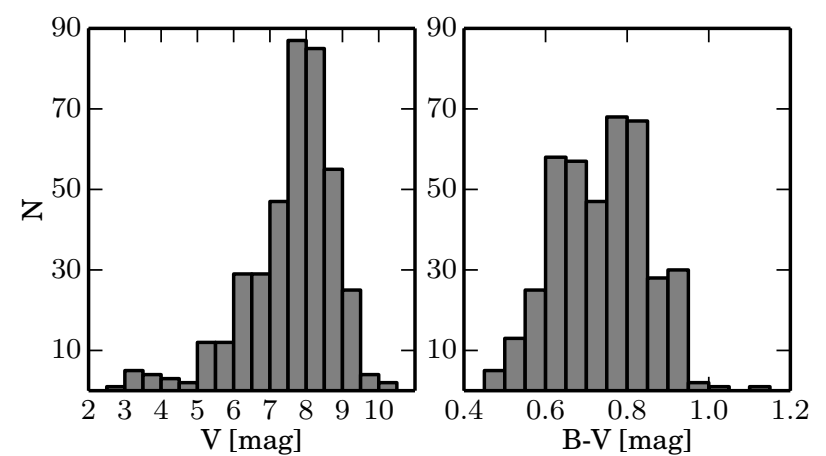

Fig. 1. Histograms of the apparent magnitudes in the $V$ band (left panel) and $(B-V)$ (right panel) for 402 stars observed within PTPS.

The collected spectra consist of 46 blue echelle orders (407-592 $\mathrm{nm}$ ) and 24 red orders (602-784 $\mathrm{nm})$. We reduced the data with a pipeline based on IRAF $^{2}$ tasks (flat fielding, wavelength calibration, and normalization to continuum). The signal-to-noise ratio was typically better than 200 per resolution element. For every star at least one so-called GC0 spectrum is available, which is a spectrum obtained without a $\mathrm{I}_{2}$ gas cell inserted into the optical path, and a series of GC1 spectra that are obtained with the gas cell. The observational material we used are the best-quality GC0 spectra and all available GC1 spectra for a sample of 455 subgiant and giant stars. The sample includes 11 SB1 systems.

The sample was designed as a blind extension of the RGC sample, which has been observed with HET (see Paper I) and consists of a set of field stars that meet several requirements. One of them is a location on the Herztsprung-Russell diagram (HRD) that corresponds to subgiants and giants. To identify these objects, we used photometric data and parallaxes from the Hipparcos and Tycho catalogs, choosing stars with $B-V=$ $0.55-0.95$ that are located in the expected $M_{V}$ range. For an efficient use of HET/HRS observing time, the selected stars are randomly distributed over the HET field of view $\left(\delta=-10^{\circ} 20^{\prime}\right.$ to $+71^{\circ} 40^{\prime}$ ). The adopted observing strategy sets a limit for the stellar brightness of the observed stars. The observed stars of PTPS should be brighter than $10.5^{\mathrm{mag}}$, and this threshold was also applied during the selection. The complete list of selected targets is presented in Table 1 (see also Fig. 1).

\section{Spectroscopic analysis}

The spectroscopic analysis included a check for presence of stellar companions with a cross-correlation technique, absolute radial RV measurements, atmospheric parameter determinations with an LTE analysis of Fe I and II lines, and rotation velocity estimates.

This approach was proved to be reliable. For three objects from Paper I, HD 102272, BD+20 2457, and BD+48 738, practically identical (within $1 \sigma$ ) atmospheric parameters were obtained by Mortier et al. (2013) (except [Fe/H] for BD+20 2457, which was found $\sim 3 \sigma$ lower). Agreement within $1 \sigma$ was also obtained for parameters of another five stars analyzed by Sousa et al. (2015): HD 17092, HD 240210, HD 240237, HD 96127 , and HD 219415.

\footnotetext{
2 IRAF is distributed by the National Optical Astronomy Observatories, which are operated by the Association of Universities for Research in Astronomy, Inc., under cooperative agreement with the National Science Foundation.
}

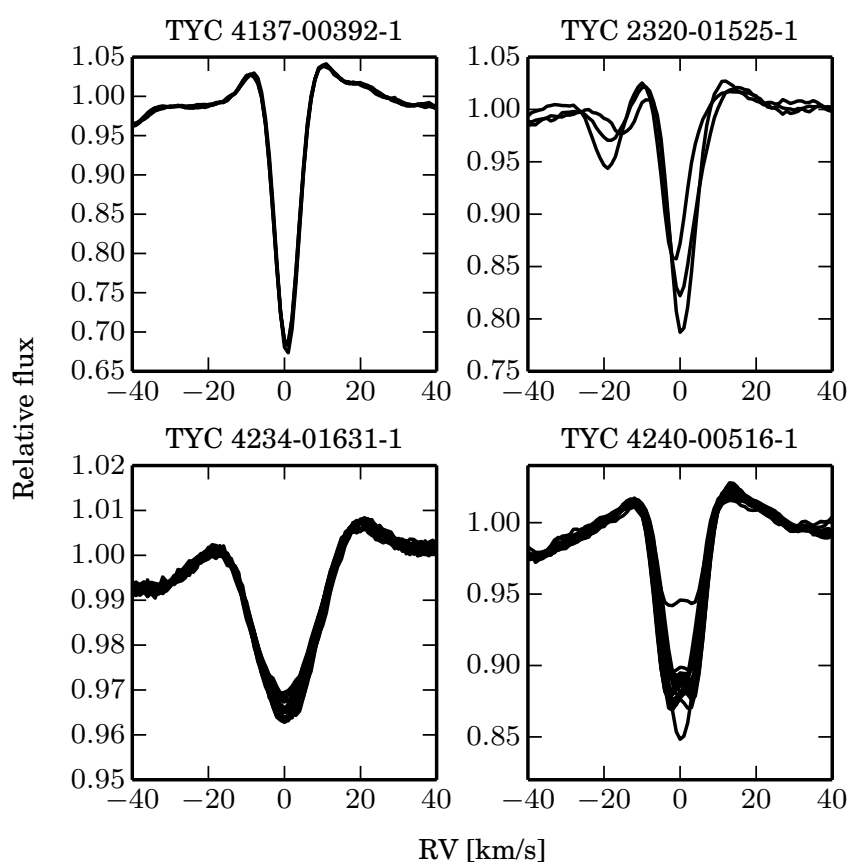

Fig. 2. Sample of CCF for four objects: top left - single star or SB1; top right - SB2; bottom left - weak line (low metallicity or fast rotator); bottom right: variable $\mathrm{CCF} / \mathrm{SB} 2$.

\subsection{Cross-correlation function analysis}

To construct the cross-correlation functions (CCFs), we correlated all available GC1 stellar spectra for each star with a numerical mask consisting of 1 and 0 value points, after cleaning the spectra from the I 2 lines with the ALICE code (Nowak 2012; Nowak et al. 2013). The non-zero points correspond to the positions of 300 unblended, isolated stellar absorption lines at zero velocity that are present in a synthetic ATLAS9 (Kurucz 1993) spectrum of a K2 star. We used only the first 17 orders of the blue spectra. They are free of telluric lines and corrected in the wavelength scale using the $\mathrm{I}_{2}$ lines. The $\mathrm{CCF}$ was computed step by step for each velocity point in a single order. For every order the algorithm selected only those lines from the mask that were suitable for a given wavelength range. CCFs from all orders were finally added to obtain the final CCF for the whole spectrum.

The shape of the CCF and its variation in the series of available spectra were used to identify spectroscopic binaries with resolved spectral line systems (SB2), objects with variable $\mathrm{CCF}$, and stars with flat CCF (fast rotators or low-metallicity stars). Typical CCFs for various cases are presented in Fig. 2. We summarize the results of the CCF analysis in Table 2.

After the preliminary analysis, some objects appeared unsuitable for a planet search as a result of the way we selected the stars for this sample; these were rejected. Fifty-three stars in total were rejected based on various conditions. Our CCF analysis revealed a group of 25 stars with multiple CCFs and 22 with unresolved and variable CCF depths. We labeled these stars spectroscopic binaries - SB2 and variable CCF/SB2, respectively. For another group of 5 stars the absorption line system was too weak to correctly measure the EW. These stars we labeled weak $\mathrm{CCF}$, and they may be fast rotators or very low metallicity stars that are likewise unsuitable for a planet search. We also excluded from the analysis one object that probably has a very low effective temperature. 

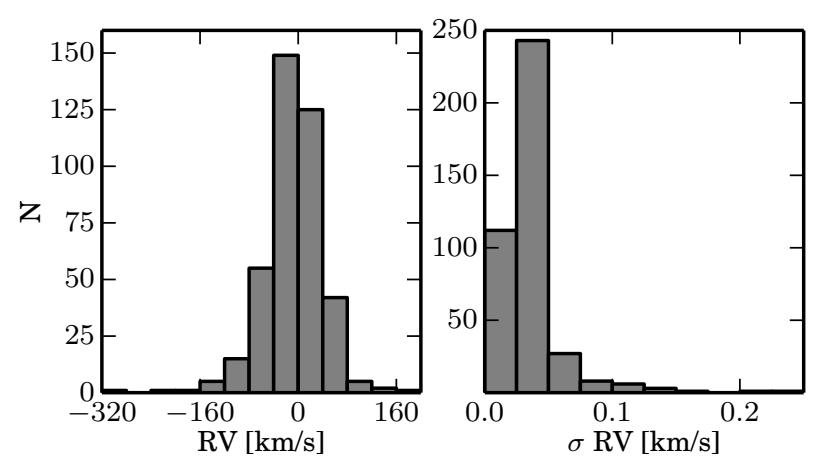

Fig. 3. Histograms of the RVs obtained from the cross-correlation function (left panel) and their uncertainties (right panel) for the PTPS stars.
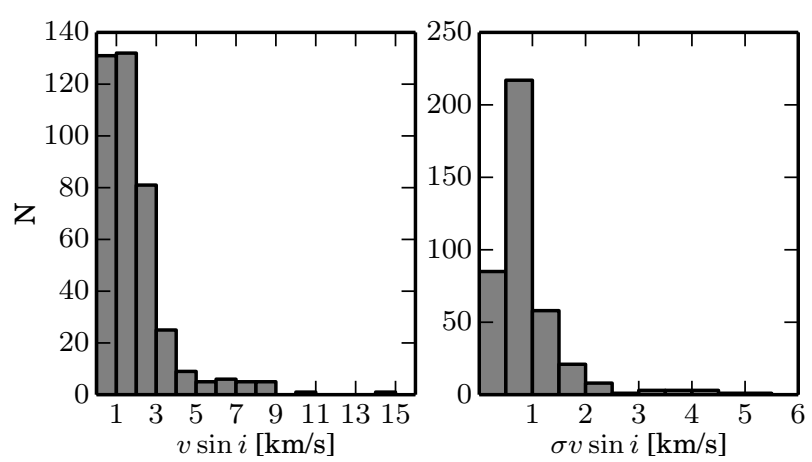

Fig. 4. Histograms of the rotational velocities and their uncertainties obtained from SME (Valenti \& Piskunov 1996) synthetic spectrum fitting.
A detailed spectroscopic analysis was not possible for these stars because their equivalent widths are misleading or accompanied with huge uncertainties. To roughly estimate atmospheric parameters for these stars in our analysis, we adopted for all of them $T_{\text {eff }}, \log g$, and initial luminosity estimates from Adamów $\&$ Niedzielski (2010), that is, values of $T_{\text {eff }}$ obtained from the empirical calibration of Ramírez \& Meléndez (2005) based on Tycho and 2MASS photometry, and $\log g$ roughly estimated using the method of Bilir et al. (2006) and Gelino et al. (2005). The results of this simplified analysis are presented in Table 2 for completeness, but they were ignored in the subsequent analysis.

\subsection{Absolute radial velocities}

The absolute radial velocity was measured by fitting a Gaussian function to the coadded CCFs for the 17 orders of the blue spectrum of the best available GC0 spectrum, obtained with the ALICE code (Nowak 2012; Nowak et al. 2013).

The uncertainty of the resulting RV was computed as $\mathrm{rms} / \sqrt{17}$ of the $17 \mathrm{RVs}$ obtained for each order separately. The mean standard uncertainty obtained in this way is $\sigma \mathrm{RV}_{\mathrm{CCF}}=$ $0.039 \mathrm{~km} \mathrm{~s}^{-1}$. However, as HET/HRS is neither thermally nor pressure stabilized, the RVs are subject to seasonal variations, and the actual precision is in the $2-3 \mathrm{~km} \mathrm{~s}^{-1}$ range. The distribution of RVs and their uncertainties is presented in Fig. 3.

Radial velocities were transformed to the barycenter of the solar system with the algorithm of Stumpff (1980) for all stars in our sample They are presented in Table 1 (Col. 11) together with the epochs of observation as modified Julian date (MJD, Col. 12).

\subsection{Rotational velocities}

We obtained projected rotational velocities, $v \sin i_{\star}$, by modeling the GC0 and red stellar spectra with the Spectroscopy Made Easy tool (SME, Valenti \& Piskunov 1996). A more detailed description of this method is presented in Paper II. Estimated rotational velocities are presented in Table 1 (Col. 10). Most of our objects are slow rotators with $v \sin i_{\star}$ of $1-3 \mathrm{~km} \mathrm{~s}^{-1}$. Only two objects show $v \sin i_{\star}$ higher than $10 \mathrm{~km} \mathrm{~s}^{-1}$ : TYC 1427-00095-1 and TYC 1515-00866-1. We show the histograms of our estimates of $v \sin i_{\star}$ and their uncertainties in Fig. 4.

\subsection{Equivalent widths}

We used the code Automatic Routine for line Equivalent widths in stellar Spectra (ARES, Sousa et al. 2007) to measure the equivalent widths (EWs) of spectral lines. The code allowed us to automatically measure EWs for a set of neutral and ionized iron absorption lines, and we found it to be more flexible than DAOSPEC (Stetson \& Pancino 2008), which we had used in Paper I. To test the consistency between ARES and DAOSPEC measurements, we compared EW measurements of Fe lines (with $E W<200 \mathrm{~m} \AA$ ) for five stars (HD 3933, 102842, 187094,215443 , and 9416) obtained with both tools. The two sets of measurements agreed very well (the Pearson correlation coefficient $r=0.943), E W_{\mathrm{DAOSPEC}}=0.911 \pm 0.017 \times E W_{\mathrm{ARES}}+$ $5.049 \pm 0.852$, with an average difference of $6.50 \pm 10.22 \mathrm{~m} \AA$ and an average ratio of $E W_{\mathrm{ARES}} / E W_{\mathrm{DAOSPEC}}=1.06 \pm 0.41$. This justified our continuing with ARES.

The selection of spectra lines used in spectroscopic analysis may lead to slight variations in the results (Tsantaki et al. 2013; Alves et al. 2015). For consistency with Paper I and to exploit the available spectra, we here chose the line list by Takeda et al. (2005) as the most adequate for our HET/HRS spectra following the results reported by Adamów et al. (2015). We removed from it all lines from regions with strong telluric lines. Finally, we included 220 lines (200 Fe I and $20 \mathrm{Fe}$ II) in the 4813-7855 range in our spectroscopic analysis.

\subsection{Atmospheric parameters}

After we obtained the EW measurements, we applied them as input data to determine the LTE atmospheric parameters of our sample stars. The $T_{\text {eff }}, \log g, v_{\mathrm{t}}$, and $[\mathrm{Fe} / \mathrm{H}]$ (defined in a standard manner as $\left.[\mathrm{Fe} / \mathrm{H}]=\log \left(N_{\mathrm{Fe}} / N_{\mathrm{H}}\right)-\log \left(N_{\mathrm{Fe}} / N_{\mathrm{H}}\right)_{\odot}\right)$ were obtained with the TGVIT code that has been developed by Takeda et al. (2002, 2005); see Paper I for a more detailed description of our implementation of the code. To calculate $[\mathrm{Fe} / \mathrm{H}]$, we adopted the $A(\mathrm{Fe})$ solar value of 7.50 dex (Kurucz 1993; Holweger et al. 1991). For 402 objects we obtained converged solutions for each of the stellar parameters typically in ten or fewer iterations.

To test the method with which we determined the atmospheric parameters, we applied it to Arcturus. We acquired several spectra for this object within PTPS and used the best-quality spectrum $(S / N=420)$. The atmospheric parameters we obtained, $T_{\text {eff }}=4254 \pm 20 \mathrm{~K}, \log g=1.61 \pm 0.08, v_{\mathrm{t}}=1.54 \pm 0.07$ and $[\mathrm{Fe} / \mathrm{H}]=-0.61 \pm 0.08$, agree very well with those from Ramírez \& Allende Prieto (2011), $T_{\text {eff }}=4286 \pm 30 \mathrm{~K}, \log g=$ $1.66 \pm 0.5$, and $[\mathrm{Fe} / \mathrm{H}]=-0.52 \pm 0.04$, and from Martin (1977), $T_{\text {eff }}=4300 \pm 90 \mathrm{~K}, \log g=1.74 \pm 0.2, v_{\mathrm{t}}=1.70$ and $[\mathrm{Fe} / \mathrm{H}]=$ $-0.51 \pm 0.08$ (see Sect. 3.6 for a comment on the uncertainty of atmospheric parameters). We are therefore confident that the applied method is correct. 

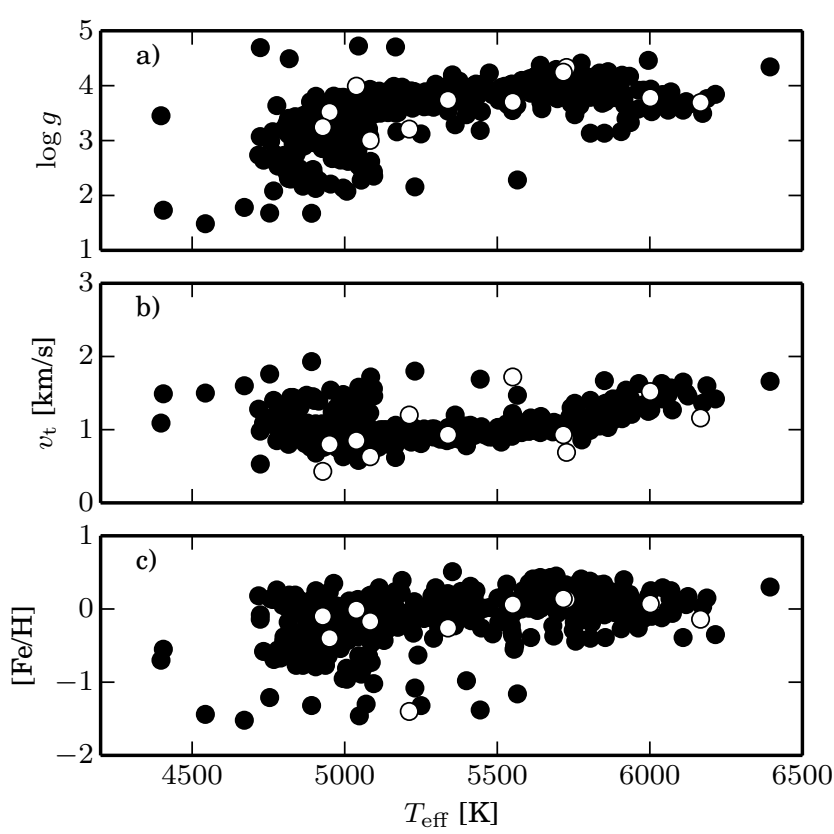

Fig. 5. Relations between $T_{\text {eff }}, \log g, v_{\mathrm{t}}$, and $[\mathrm{Fe} / \mathrm{H}]$ for 402 stars with complete spectroscopic analysis. Results for $11 \mathrm{SB} 1$ are shown as open circles.

The atmospheric parameters we determined for 402 GK stars are presented in Table 1 (Cols. 6-9), where the numerical values of $T_{\mathrm{eff}}, \log g, v_{\mathrm{t}}$, and $[\mathrm{Fe} / \mathrm{H}]$ are presented together with their intrinsic uncertainties.

The values of resulting atmospheric parameters generally remain within the range of the TGVIT model grids.

Figure 5 shows the relations between the atmospheric parameters presented in Table 1.We note that the results obtained for single-line spectroscopic binaries (SB1) agree very well with those for apparently single stars.

The effective temperature $T_{\text {eff }}$ lies between $4398 \mathrm{~K}$ and $6394 \mathrm{~K}$ with a median value of $5167 \mathrm{~K}$. The distribution of $T_{\text {eff }}$, presented in Fig. 6a, shows two maxima, one for giants (cooler) and one for subgiants (warmer), which are the two most numerous groups of stars in our sample. The intrinsic uncertainty distribution in $T_{\text {eff }}$ is presented in Fig. 6e.

The gravitational accelerations $\log g$ for our sample range between 1.49 and 4.72 with a median of 3.65. The distribution of $\log g$ is presented in Fig. 6b. We found that 5 stars have $\log g<$ 2.0, 60 have $3.0>\log g \geq 2.0,277$ have $4.0>\log g \geq 3.0$, and 60 stars have $\log g \geq 4$.0. Most of our stars (194) have $\log g$ of 3.5-4.0, which means that generally they are subgiants. The uncertainty distribution in $\log g$ is presented in Fig. 6f.

The microturbulence velocity, $v_{\mathrm{t}}$, reaches values from $0.43 \mathrm{~km} \mathrm{~s}^{-1}$ to $1.93 \mathrm{~km} \mathrm{~s}^{-1}$ and has a median at $1.03 \mathrm{~km} \mathrm{~s}^{-1}$. Most of our stars have $v_{\mathrm{t}}$ between $0.8 \mathrm{~km} \mathrm{~s}^{-1}$ and $1.2 \mathrm{~km} \mathrm{~s}^{-1}$ (Fig. 6c). The uncertainty distribution in $v_{\mathrm{t}}$ is presented in Fig. 6g.

The metallicity of the stars in our sample, $[\mathrm{Fe} / \mathrm{H}]$, remains within the limit of -1.52 to +0.51 , with a median value at -0.08 . Most of our objects have $[\mathrm{Fe} / \mathrm{H}]$ in the range of -0.25 to 0.25 . Figure $6 \mathrm{~d}$ presents the distribution, which clearly shows that our stars are mostly less metal abundant than the Sun. The uncertainty distribution in $[\mathrm{Fe} / \mathrm{H}]$ is presented in Fig. $6 \mathrm{~h}$.

\subsection{Uncertainy estimates of the atmospheric parameters}

The mean intrinsic uncertainties of our determinations, that is, those delivered by TGVIT, are $\sigma T_{\text {eff }}=15 \mathrm{~K}, \sigma \log g=0.04$, $\sigma v_{\mathrm{t}}=0.07 \mathrm{~km} \mathrm{~s}^{-1}$, and $\sigma[\mathrm{Fe} / \mathrm{H}]=0.02$. Figure 7 shows the intrinsic uncertainties of these three parameters as a function of $T_{\text {eff }}$. No correlation exists between the uncertainties and the obtained parameter values for any of the atmospheric parameters in the wide range of $T_{\text {eff }}$ between $4500 \mathrm{~K}-6000 \mathrm{~K}$. The scatter of the uncertainties is uniformly distributed over the whole range of resulting parameters. We note, however, that for stars with $T_{\text {eff }}$ below $4500 \mathrm{~K}$ or above $6000 \mathrm{~K}$ the uncertainties are slightly higher, especially in $T_{\text {eff }}$ and $[\mathrm{Fe} / \mathrm{H}]$.

To test the effect of the shift in EWs between DAOSPEC and ARES measurements presented in Sect. 3.4, we calculated stellar atmospheric parameters for the same five stars using DAOSPEC and ARES EWs separately. We obtained agreement within $1 \sigma$ intrinsic in all parameters except for the microturbulence velocity, in which the results differ by 2-3 intrinsic $\sigma$. We therefore assume that our results here are consistent with those of Paper I.

All these intrinsic uncertainties, except for $[\mathrm{Fe} / \mathrm{H}]$, are numerical uncertainties resulting from the iterative procedures of TGVIT, which only represent a goodness of fit, however. The intrinsic uncertainties in metallicities are estimated in TGVIT from the actual $\mathrm{Fe}$ abundance distribution as the standard deviation of the mean. A detailed comparison with Soubiran et al. (1998) and Butler et al. (2006) presented in Paper I suggests that our intrinsic uncertainties in $T_{\text {eff }}, \log g$, and $v_{\mathrm{t}}$ are underestimated by a factor of 2-3. In Table 1 we present the intrinsic uncertainties from TGVIT, allowing for a future more detailed uncertainty analysis.

Both atmospheric parameters and their uncertainties for SB1 stars in the presented sample fit the general trends for single stars well (Figs. 5 and 7), and therefore we address single and SB1 stars together below.

\subsection{Comparison with the literature}

The atmospheric parameters for 272 of the stars discussed here were determined for the first time.

In our attempt to compare our results with those available in the PASTEL catalog (Soubiran et al. 2010), we found 130 stars in common, many with multiple records in PASTEL, which sometimes results in large scatter. After removing the objects with most discrepant values from the comparison (labeled in Fig. 8), good agreement was found for all parameters.

For $T_{\text {eff }}$ (444 measurements in the PASTEL catalog) the Pearson correlation coefficient is $r=0.96$ (Fig. 8a), the mean difference between measurements $\delta=87 \pm 86 \mathrm{~K}$, and on average the ratio of our to their results is $\rho=1.00 \pm 0.02$.

For $\log g$ (244 records in the PASTEL catalog) we also found a very good agreement, with $r=0.92, \delta=0.19 \pm 0.15$, and $\rho=1.02 \pm 0.08$ (Fig. 8b).

For $[\mathrm{Fe} / \mathrm{H}]$ (248 records in the PASTEL catalog), we found $r=0.95, \delta=0.09 \pm 0.08$, and $\rho=0.75 \pm 2.47$ (Fig. 8c). Technically, this result suggests that our $[\mathrm{Fe} / \mathrm{H}]$ determinations are systematically lower than those of the PASTEL catalog. This result is misleading, however, and is caused by a scatter in results for $|[\mathrm{Fe} / \mathrm{H}]|<0.1$, which usually is consistent within the uncertainties. For 181 records with $|[\mathrm{Fe} / \mathrm{H}]|>0.1$ we found $\rho=0.93 \pm 0.5$.

We compare in Fig. 8d the RVs obtained here with the CCF technique with literature data for 279 objects included in the 

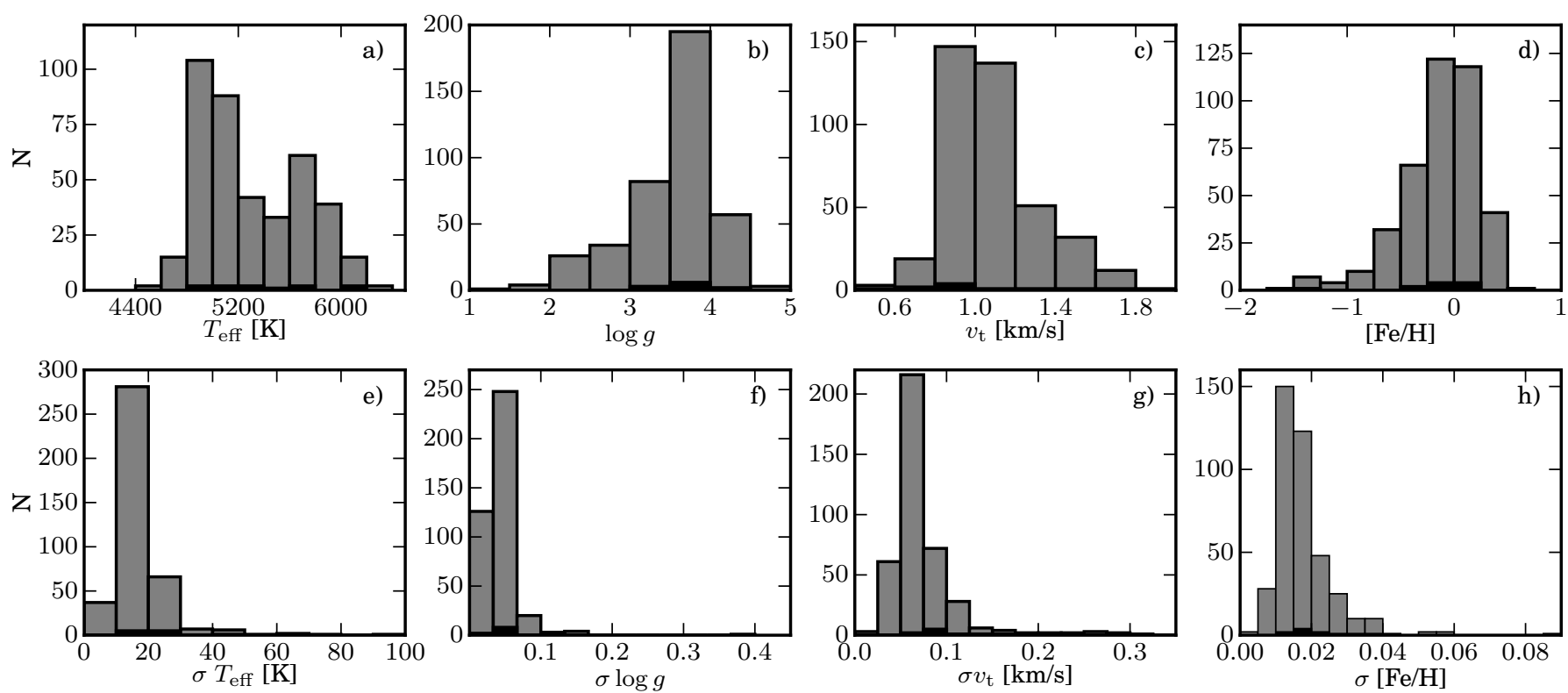

Fig. 6. Histograms of of $T_{\text {eff }}, \log g, v_{\mathrm{t}}$, and [Fe/H] obtained for 402 stars with complete spectroscopic analysis. Results for $11 \mathrm{SB} 1$ are shown in black.
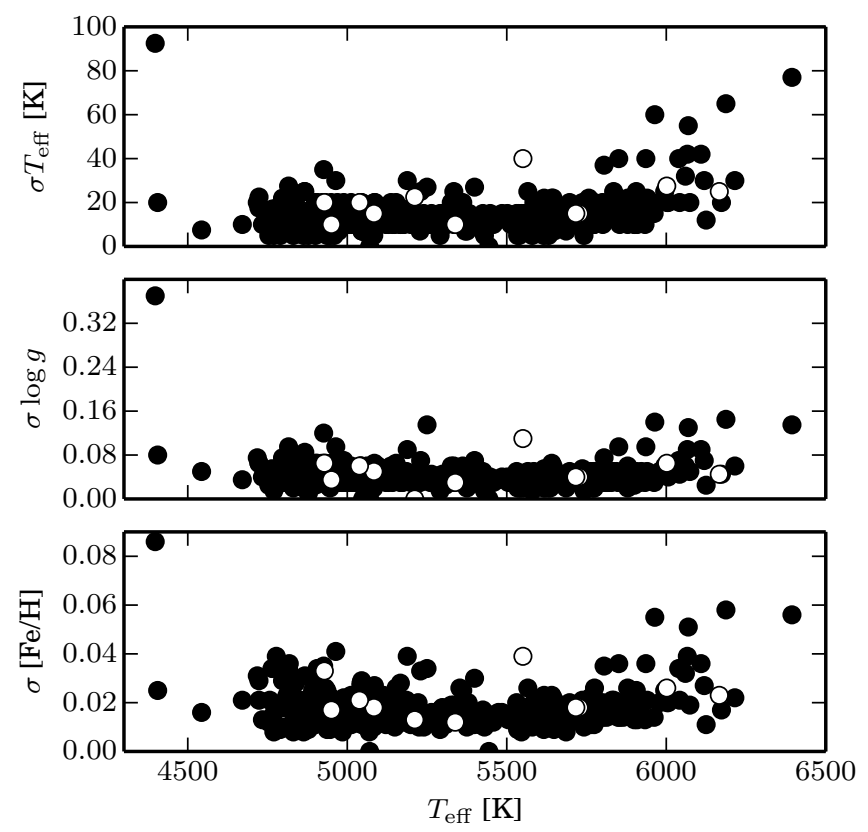

Fig. 7. Relations between intrinsic uncertainties of $\sigma T_{\text {eff }}$ (top panel), $\sigma \log g$ (middle panel), and $\sigma[\mathrm{Fe} / \mathrm{H}]$ (bottom panel) vs. $T_{\text {eff }}$ for 402 stars. Results for 11 SB1 are shown as open circles.

SIMBAD $^{3}$ database. We found $r=0.97, \delta=1.8 \pm 3.2 \mathrm{~km} \mathrm{~s}^{-1}$, and $\rho=0.98 \pm 0.92$ ).

No systematic effects were found. Our results agree with those obtained by other authors within the estimated uncertainties.

\section{Luminosities, masses, ages, and radii}

We estimated an intrinsic color index $(B-V)_{0}$ and bolometric corrections $B C_{\mathrm{V}}$ for our stars using the empirical calibrations

\footnotetext{
3 The SIMBAD astronomical database is operated at the CDS in Strasbourg, France.
}

by Alonso et al. (1999). For 374 stars with available Hipparcos parallaxes (van Leeuwen 2007) assuming the standard interstellar reddening with a total-to-selective extinction ratio $R_{V}=3.1$ (Rieke \& Lebofsky 1985) luminosities were directly calculated. These luminosities were used in the subsequent analysis.

\subsection{Stellar masses and ages}

Stellar masses $M / M_{\odot}$ and estimates of ages were obtained with the Adamczyk et al. (2015) modification of the Bayesian method based on the formalism developed by Jørgensen \& Lindegren (2005), which has been modified by da Silva et al. (2006) to avoid statistical biases and to take the uncertainty estimates of the observed quantities into consideration. We adopted theoretical stellar models from Bressan et al. (2012) and used isochrones with intervals of $Z=0.0001,0.0004,0.0008,0.001,0.002$, $0.004,0.006,0.008,0.01,0.0152,0.02,0.025,0.03,0.04,0.05$, 0.06 , and 0.008 in log (age/yr). The adopted solar distribution of heavy elements corresponds to the solar metallicity $Z \simeq 0.0152$ (Caffau et al. 2011). The helium abundance for a given metallicity was obtained from the relation $Y=0.2485+1.78 Z$ (Bressan et al. (2012). For most of our targets with Hipparcos parallaxes available, we used $T_{\text {eff }}, \log g,[\mathrm{Fe} / \mathrm{H}]$, and the luminosity as input parameters for the Bayesian analysis. For stars without a reliable parallax we were able to obtain luminosity estimates from the Bayesian analysis as well.

A detailed description of this approach is presented in Adamczyk et al. (2015).

The resulting masses range from $0.52 M_{\odot}$ to $3.21 M_{\odot}$, as the histogram of $M / M_{\odot}$ for all our stars presents (Fig. 9b). Most of our stars have masses of up to $1.19 M_{\odot}$ (median). However, there is a significant number $(17$ or $\sim 4.2 \%$ ) of stars in the intermediate-mass range $2 M_{\odot} \leq M \leq 3.21 M_{\odot}$. Table 1 (Col. 17) presents the final adopted masses.

The uncertainties of the stellar masses that were obtained by comparison with theoretical isochrones depend on the accuracy with which the star's location in the $\left[\log L / L_{\odot}, \log g, \log T_{\text {eff }}\right]$ space is determined. 

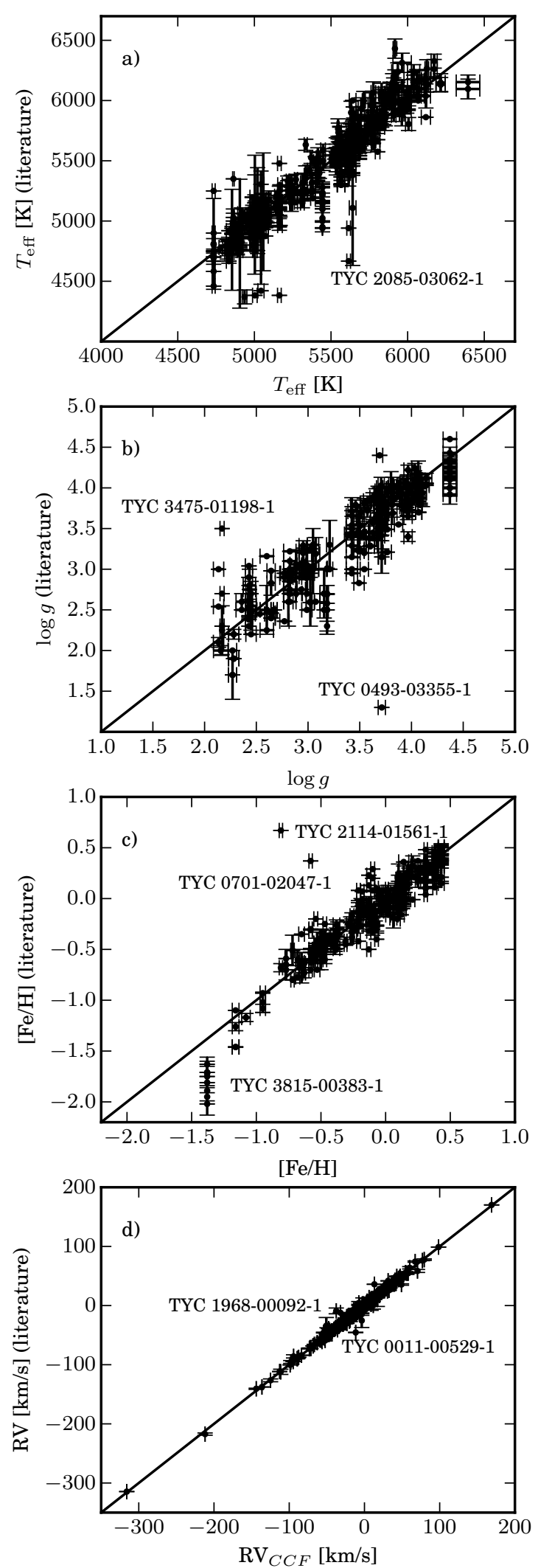

Fig. 8. Comparisons between our $T_{\text {eff }}, \log g$, [Fe/H], and literature values from the PASTEL catalog (Soubiran et al. 2010). Radial velocities derived from our cross-correlation analysis are compared with measurements available in SIMBAD. The RV uncertainties are presented when available. The solid lines present the one-to-one relations.

The main source of the uncertainty in mass is the parallax, or luminosity, and its uncertainties. For stars whose parallax was more precise, the simplified treatment of metallicity may introduce an additional uncertainty in mass through the choice of the metallicity model and then isochrone. We found that the mean uncertainty for the stellar mass is $\sigma M / M_{\odot}=0.07$ for the whole sample. However, uncertainties may become much larger for very confusing isochrones at lower temperatures $\left(T_{\mathrm{eff}} \leq\right.$ $4500 \mathrm{~K})$. We also note that for stars from the RGC, which may undergo stellar mass-loss, the masses obtained this way are upper limits because in Bressan et al. (2012) the stellar mass lost due to mass loss is added while constructing the isochrones, and the MS masses are given.

Estimated stellar ages are presented in Table 1 (Col. 19) and in Fig. 9c. We found that typical stars from our sample have $\log (\mathrm{age} / \mathrm{yr})$ between 9.5 and 10 and have mean uncertainties around 0.09 .

\subsection{Stellar luminosities}

For 28 stars without parallaxes (or $\sigma_{\pi}>\pi$ ), the luminosity was adopted from fits to the isochrones, corresponding to the determined stellar mass. In Table 1 (Col. 15) we list the final adopted luminosity. The $\log L / L_{\odot}$ ranges from -1.25 to 2.84 with a median of 0.76 (Fig. 9a).

For stars with luminosities calculated from Hipparcos parallaxes, we calculated the luminosity uncertainties using the standard exact differential law for calculations based on the available $\pi$ and photometry uncertainties. For Bayesian estimates they were estimated as the dispersion of the respective PDF (Fig. 10) for the proper stellar mass. The average value of the luminosity uncertainty for the whole sample is $\sigma \log L / L_{\odot}=0.14$.

In Fig. 11 we present a comparison of stellar luminosities obtained from Hipparcos parallaxes $\left(\sigma_{\pi}<\pi\right)$ and from our Bayesian analysis based on atmospheric parameters alone. A general agreement is clear, although for stars with very small parallaxes ( $\pi \lesssim 10$ mas) both estimates may vary substantially. In Fig. 11 we also present (in red) luminosities obtained with the two methods for the Paper I stars by Adamczyk et al. (2015). This sample contains stars at greater distances, for which the luminosity is certainly less reliable.

\subsection{Stellar radii}

With either spectroscopic $\log g$ and adopted stellar mass or spectroscopic $T_{\mathrm{eff}}$ and adopted luminosities, we were able to calculate stellar radii (see Adamczyk et al. 2015 for more details). Although for several stars the radii obtained from these two sets of parameters differ significantly, the general agreement between both $R / R_{\odot}$ estimates is good $(r=0.93)$. The maximum uncertainties were estimated in both approaches by applying the standard exact differential law.

Figure $9 \mathrm{~d}$ shows that the radii range from $0.66 R_{\odot}$ to $36.04 R_{\odot}$. Most of our stars have radii of about $0.66-4 R_{\odot}$. The median and mean $R / R_{\odot}$ was found to be 2.76 and 4.24 , respectively. On average, the precision is $\sigma R / R_{\odot}=0.19$. The adopted stellar radii are presented in Table 1(Col. 18).

The ranges of adopted uncertainties of radii as well as luminosities, masses, and ages are depicted in Fig. 9 (panels e-h).

\section{PTPS sample of evolved stars}

The PTPS sample of evolved starst consists of the 402 stars presented in this paper together with the 342 stars from the RGC sample discussed in Paper I with revised, integrated parameters 

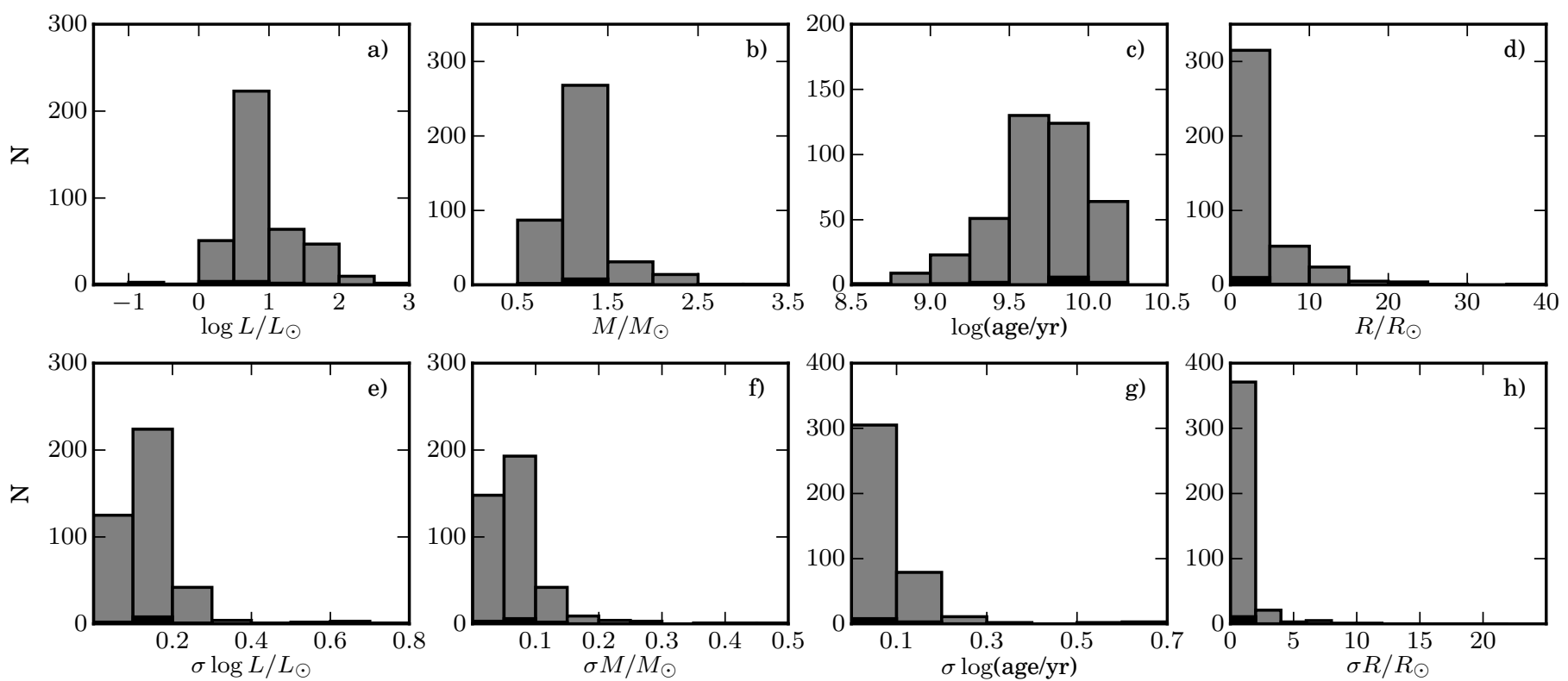

Fig. 9. Distributions of $\log L / L_{\odot}, M / M_{\odot}, \log \left(\right.$ age/yr), and $R / R_{\odot}$ for 402 stars with complete spectroscopic analysis (panels a)-d)). The histograms for the uncertainty estimates are presented in panels $\mathbf{e})-\mathbf{h}$ ). Results for 11 SB1 are shown in black.

presented in Adamczyk et al. (2015). For all 744 stars we obtained the atmospheric parameters as well as masses, luminosities, ages, and radii in a uniform way. The two subsamples are complementary, despite differences resulting from their definitions. The main difference between them is that giants and distant bright giants are mostly present in the sample of Paper I, while subgiants are highly abundant in the sample presented here. Another difference is the amount of stars with available Hipparcos parallaxes. In contrast to the sample presented in Paper I, parallaxes are available for most stars discussed here. As a result, luminosity, masses, and ages estimates presented here are presumably more precise (cf. Adamczyk et al. 2015). In Fig. 10 we present the HRD for the complete evolved sample of the PTPS stars, together with a subsample of dwarfs that are not discussed in this paper.

We applied the approach developed by Johnson \& Soderblom (1987) to constrain the space velocity components $(U, V, W)$ for the PTPS sample of evolved stars. Following the criteria reported by Ibukiyama \& Arimoto (2002), we assigned our objects to galactic populations. Stars from thin-disk, thickdisk, and halo populations have $V>-62 \mathrm{~km} \mathrm{~s}^{-1},-182 \leq V<$ $-62 \mathrm{~km} \mathrm{~s}^{-1}$, and $V<-182 \mathrm{~km} \mathrm{~s}^{-1}$, respectively. Objects with $V>-62 \mathrm{~km} \mathrm{~s}^{-1}$ but with a distance from the Galactic plane $z>600$ pc are classified as thick-disk stars; see Fig. 12.

Most of our objects belong to the thin-disk population (over $64 \%$ ), more than $33 \%$ stars are thick-disk stars, and a few percent of the objects belong to the Galactic halo. Stars in the joint sample are generally less metal abundant than the Sun, with a median of $[\mathrm{Fe} / \mathrm{H}]=-0.12$. However, the median for each population is different: $[\mathrm{Fe} / \mathrm{H}]=-0.1$ for thin-disk stars, $[\mathrm{Fe} / \mathrm{H}]=-0.16$ for thick -disk stars, and $[\mathrm{Fe} / \mathrm{H}]=-0.90$ for halo stars.

With a substantial number of stars in both the thin- and thickdisk polpulation, the sample is also suitable for future analyses of planetary systems in a wider Galactic perspective.

\section{Evolved stars in other planet search projects}

To our knowledge, the PTPS sample of evolved stars is the largest existing sample of stars beyond the MS that have been searched for low-mass companions with a high-precision RV technique. This means that it constitutes an interesting comparison to several other samples of stars that have been searched for planets. In Fig. 10 we show that the PTPS sample of evolved stars covers a wide range of effective temperatures, between 4000 and $6500 \mathrm{~K}$, a luminosity range over three orders of magnitude, $\log L / L_{\odot}$ between -1 and $3, \log g$ ranging from 1 to 5 , and a metallicity from 0.5 down to -1.5 . It contains stars from both subgiant and red giant branches, as well as a subset of highly evolved stars in the horizontal branch or early AGB; see Fig. 10. The distribution of $\log g,[\mathrm{Fe} / \mathrm{H}]$, and masses of stars in the sample is presented in Fig. 13.

To put our sample in perspective, we collected available atmospheric and stellar parameters for evolved stars from several other planet search projects. This way, we can compare our sample with another five planets search projects: the Okayama Planet Search (Sato et al. 2003) and its extension EAPSNet, which provide 488 stars in total for which stellar parameters are available in Takeda et al. (2008), Liu et al. (2010), Wang et al. (2011); the Lick K-giant Survey (Frink et al. 2002), which lists stellar parameters for 359 stars presented in Reffert et al. (2014); the Retired A Stars and Their Companions (Johnson et al. 2007) survey, with preliminary stellar parameters for 244 stars available from Ghezzi \& Johnson (2015); and the radial velocity survey of 164 bright $\mathrm{G}$ and $\mathrm{K}$ giant stars in the southern hemisphere, EXPRESS (Jones et al. 2011). These surveys together contain 1255 stars. For all these stars stellar atmospheric parameters and stellar masses, determined in various ways, are available in the respective papers. We also added the Coralie and HARPS searches (Lovis \& Mayor 2007) with 257 their stars to the analysis. For these, atmospheric parameters (but no stellar masses) are available in Alves et al. (2015).

A comparison of the basic properties of these star samples is presented in Fig. 13, which easily shows the similarities and differences. From Fig. 13a it is clear that our sample of evolved stars covers a much wider range of stellar hosts than any other project, from bright giants to subgiants. Most of the other samples cover the evolved star range down to $\log g=1.5$, except for the Lick K-giant Survey of Reffert et al. (2014), which extends 
A. Niedzielski et al.: The Penn State - Toruń Centre for Astronomy Planet Search stars. III.

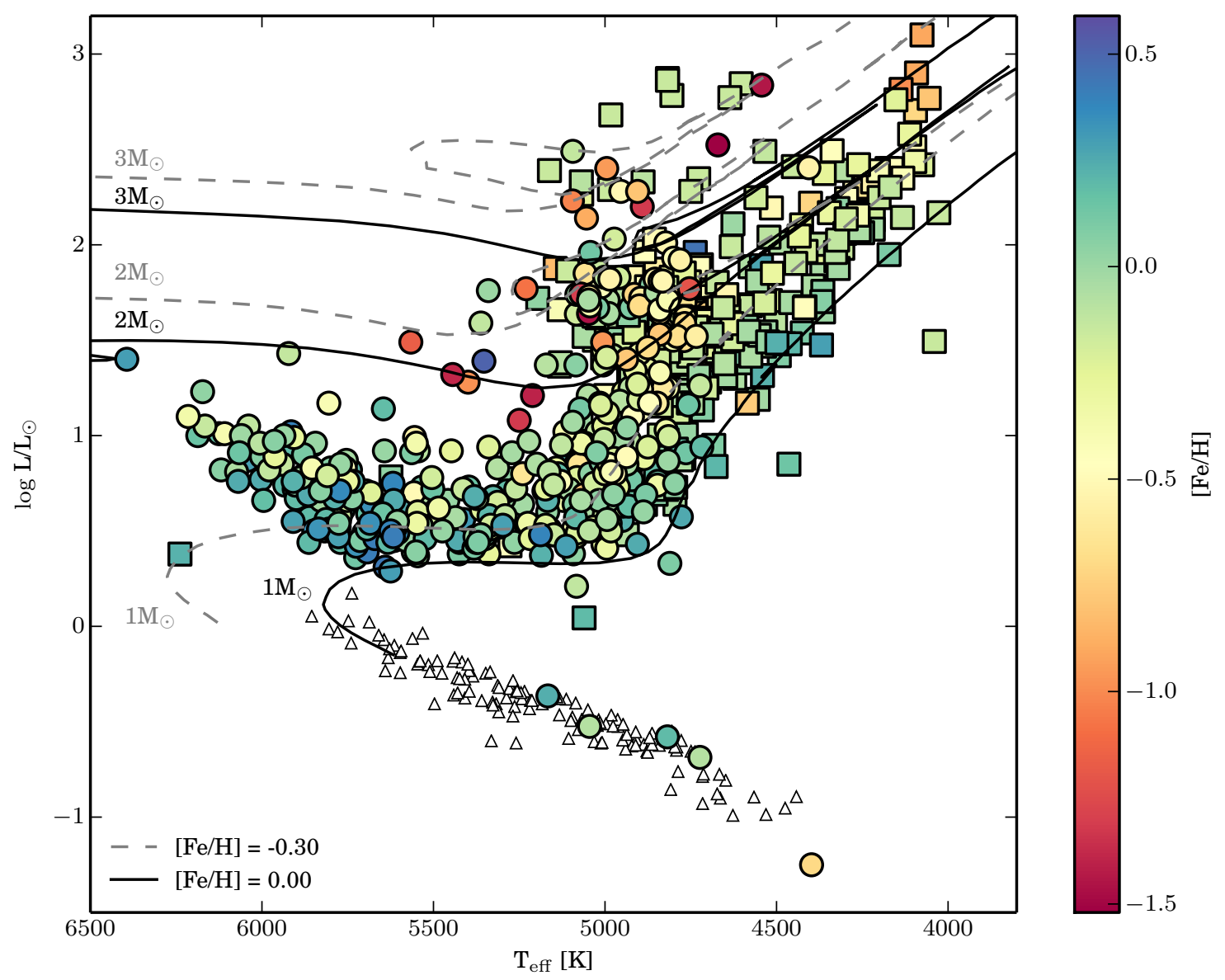

Fig. 10. H-R diagram for the complete PTPS sample of evolved stars. The stars presented here are displayed as circles, objects discussed in Paper I as squares. We also present preliminary results for dwarfs (open triangles, Deka-Szymankiewicz et al., in prep.) that were also included in PTPS, for completeness. Theoretical evolutionary tracks (Bertelli et al. 2008, 2009) are presented for stellar masses of 1-3 $M_{\odot}$ and two metallicities: the solid and dashed lines correspond to $[\mathrm{Fe} / \mathrm{H}]=-0.3$ and 0.3 , respectively. The stellar metallicity is color-coded.

to bright giants and super giants with $\log g$ as low as 0.5 . In most samples, however, the largest number of stellar hosts present $\log g=2.5-3.0$. The Retired A Stars and Their Companions sample (Ghezzi \& Johnson 2015) presents a sample that is complementary to most other surveys and is well consistent with our sample. The $T_{\text {eff }}$ distribution with most stars in 4750-5000 K range is very similar, again with the exception of the sample of Reffert et al. (2014), which extends to effective temperatures of $3750 \mathrm{~K}$.

Another important feature of the samples compared here is the very similar $[\mathrm{Fe} / \mathrm{H}]$ distribution in all surveys except for that of Jones et al. (2011), which apparently preferentially contains super-solar [Fe/H] objects (Fig. 13b).

In Fig. 13c we compare the distributions of the stellar host masses in all six surveys. Compared to our sample and the Retired A Stars and Their Companions (Ghezzi \& Johnson 2015), those of EAPSNet (Takeda et al. 2008) and the Lick K-giant Survey (Reffert et al. 2014) contain many more intermediate-mass $\left(M / M_{\odot}=2-5\right)$ stars, mostly in the $M / M_{\odot}=2-3$ range. The figure suggests that the samples were defined to preferentially contain stars in this mass range. A quick comparison of the mass distribution in Takeda et al. (2008), Liu et al. (2010), Wang et al. (2011), Reffert et al. (2014) and our samples proves that they are especially abundant in $2-3 M / M_{\odot}, 2-3 M / M_{\odot}$ and $1-2 M / M_{\odot}$, making them complementary to other planet searches around evolved stars and potentially effective in searches for planets around stars more massive than the Sun.

In Table 3 we present a compilation of the basic parameters for 62 PTPS stars that are also included in other surveys. We note the good agreement in atmospheric parameters, as illustrated in Fig. 14. However, the agreement in stellar masses is poor.

To consider the stellar masses presented in various searches in more detail, we calculated masses for all 1255 targets from all five surveys with the same method as we used for our data (Adamczyk et al. 2015) and compared them to those presented in the respective survey definition papers. The results are presented in Fig. 15. We note the good agreement with Ghezzi \& Johnson (2015) and the very good agreement with the results of Reffert et al. (2014) in Fig. 15a, b. For the stellar masses reported by Reffert et al. (2014), our values agree with their original results within $1 \sigma$ on average, with $M_{\mathrm{R}} / M_{\text {our }}=1.04 \pm 0.07$ (with a Parsons correlation coefficient of $r=0.977$ ). A similar agreement between our data and those of Ghezzi \& Johnson (2015) is apparent, with $M_{\mathrm{G}} / M_{\mathrm{our}}=1.05 \pm 0.29$, that is, with a noticeably larger scatter $(r=0.81)$.

In all other cases the stellar masses derived by us are noticeably lower. For the sample of Takeda et al. (2008) we have $M_{\mathrm{T}} / M_{\text {our }}=1.29 \pm 0.24(r=0.911)$, for that of Wang et al. (2011) $M_{\mathrm{W}} / M_{\text {our }}=1.28 \pm 0.15(r=0.948)$, for the sample of Liu et al. (2010) $M_{\mathrm{L}} / M_{\text {our }}=1.43 \pm 0.24(r=0.851)$, and for that of Jones et al. (2011) $M_{\mathrm{J}} / M_{\text {our }}=1.15 \pm 0.10(r=0.941)($ Figs. $15 \mathrm{c}-\mathrm{f})$. 

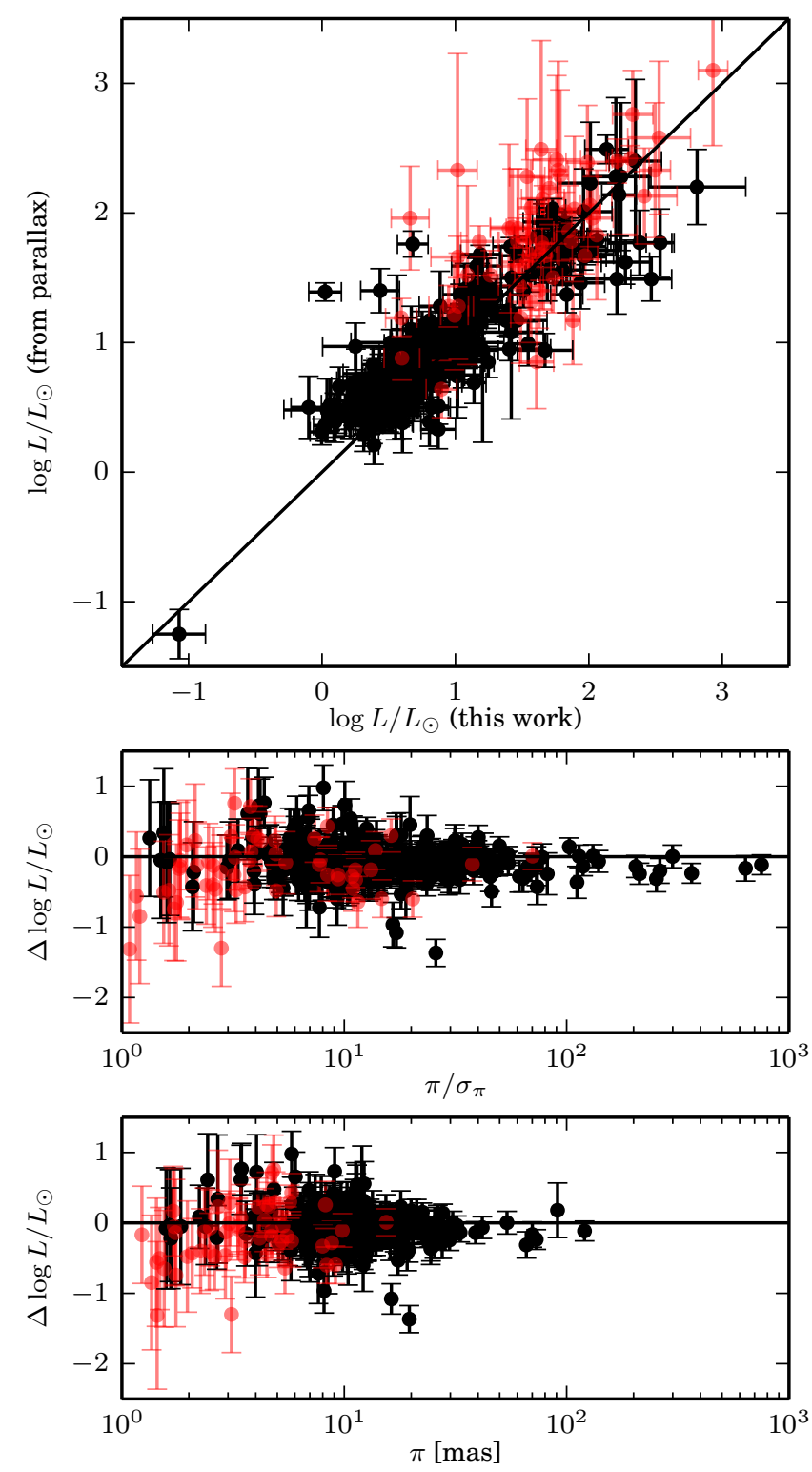

Fig. 11. Comparison of stellar luminosities obtained from HIPPARCos parallaxes and from our Bayesian analysis. The stars presented here are represented by black, those of Paper I by red symbols. The solid line presents the one-to-one relation.

The difference between ours and the published stellar masses is in some cases quite large, on average.

We note in addition that the procedure that we used to estimate stellar masses is based on isochronse of Bressan et al. (2012) and assumes a very moderate Reimers mass-loss with $\eta=0.2$, which amount was added to the resulting final stellar mass. In this approach the resulting stellar masses are MS masses, but as the estimated effect of mass-loss is low (Miglio et al. 2012), below the estimated uncertainties, the simplification is justified. The stellar mass we estimated is already most likely upper limits, however, which in turn means that the stellar mass estimates of the other samples of Takeda et al. (2008), Liu et al. (2010), Jones et al. (2011), Wang et al. (2011) are probably overestimated.

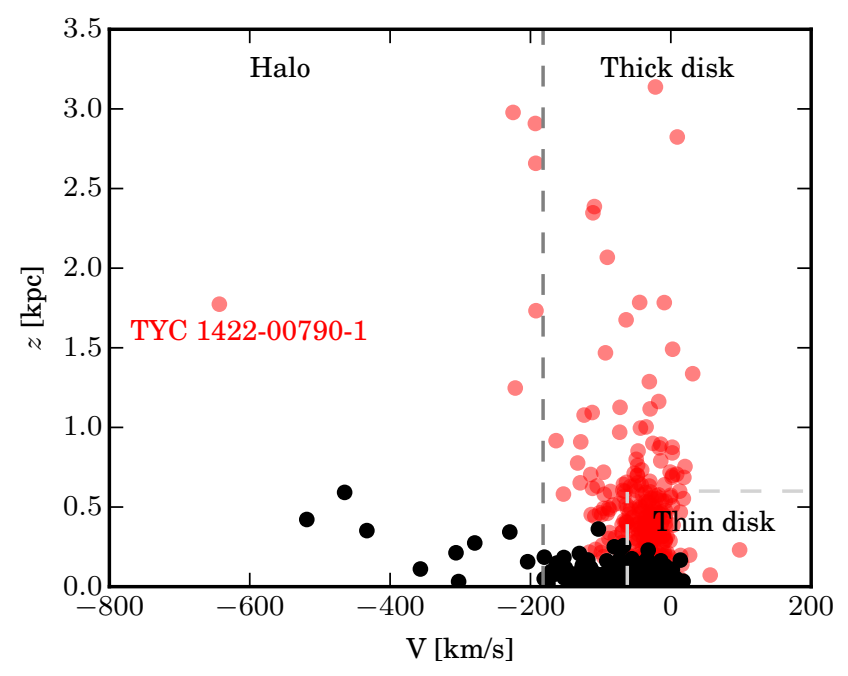

Fig. 12. Galactic rotational velocities of stars with respect to the Galactic center versus distances from the Galactic plane $z$. The stars presented here are represented by black, those of Paper I by red symbols.

\section{Discussion. Do evolved stars host more massive planets?}

With our sample and the available data on other samples of evolved stars that were searched for planets, we are in position to discuss one of the most intriguing features of the currently available sample of exoplanets around stars that are past the MS: the suspicious growth of planetary masses with evolutionary stage of theirs host (Niedzielski et al. 2015b).

Planetary systems around evolved stars are often mixed with planetary systems around stars more massive than the Sun. Although generally not true, this is somewhat justified with the increase (Niedzielski et al. 2015b) of average host mass from dwarfs $\left(M / M_{\odot}=0.997 \pm 0.016\right)$, through subgiants $\left(M / M_{\odot}=\right.$ $1.446 \pm 0.031)$, to giants $\left(M / M_{\odot}=1.885 \pm 0.091\right)$ or bright giants $\left(M / M_{\odot}=1.464 \pm 0.12\right)$. The stellar mass increase for evolved stars with planets is not a physical phenomenon, and it only reflects selection effects caused by the most important scientific driver for planet searches beyond the MS - the search for planets around stars more massive than the Sun. A real effect, postulated by Lovis \& Mayor (2007) and supported by the theory of Bowler et al. (2010) might be an increase of planetary system mass with stellar mass. This is more difficult to prove (Niedzielski et al. 2015b), however, to a great extent because of the uncertain masses of the hosts.

The question of the reliability of the masses of evolved stars hosting planetary systems was raised by Lloyd (2011, 2013), Schlaufman \& Winn (2013) and resulted in further studies (Sousa et al. 2015; Adamczyk et al. 2015). Meanwhile, Mitchell et al. (2013) and Niedzielski et al. (2013) noted that the frequency of brown dwarf (BD) companions to evolved stars seems to be surprisingly high, suggesting that there is no BD desert around these stars. Niedzielski et al. (2015b) showed that the average mass of a companion (according to exoplanet.eu) increases (RV-detected companions only) from $2.25 \pm 0.1 M_{\mathrm{J}}$ around dwarfs, through $2.956 \pm 0.467 M_{\mathrm{J}}$ around subgiants, up to $6.533 \pm 1.00 M_{\mathrm{J}}$ around giants and $7.798 \pm 1.186 M_{\mathrm{J}}$ around bright giants. This threefold increase in companion mass during the stellar evolution is probably not real (Duncan \& Lissauer 1998). It is also much faster than the average mass increase of the host of these planetary systems in these types of stars. 

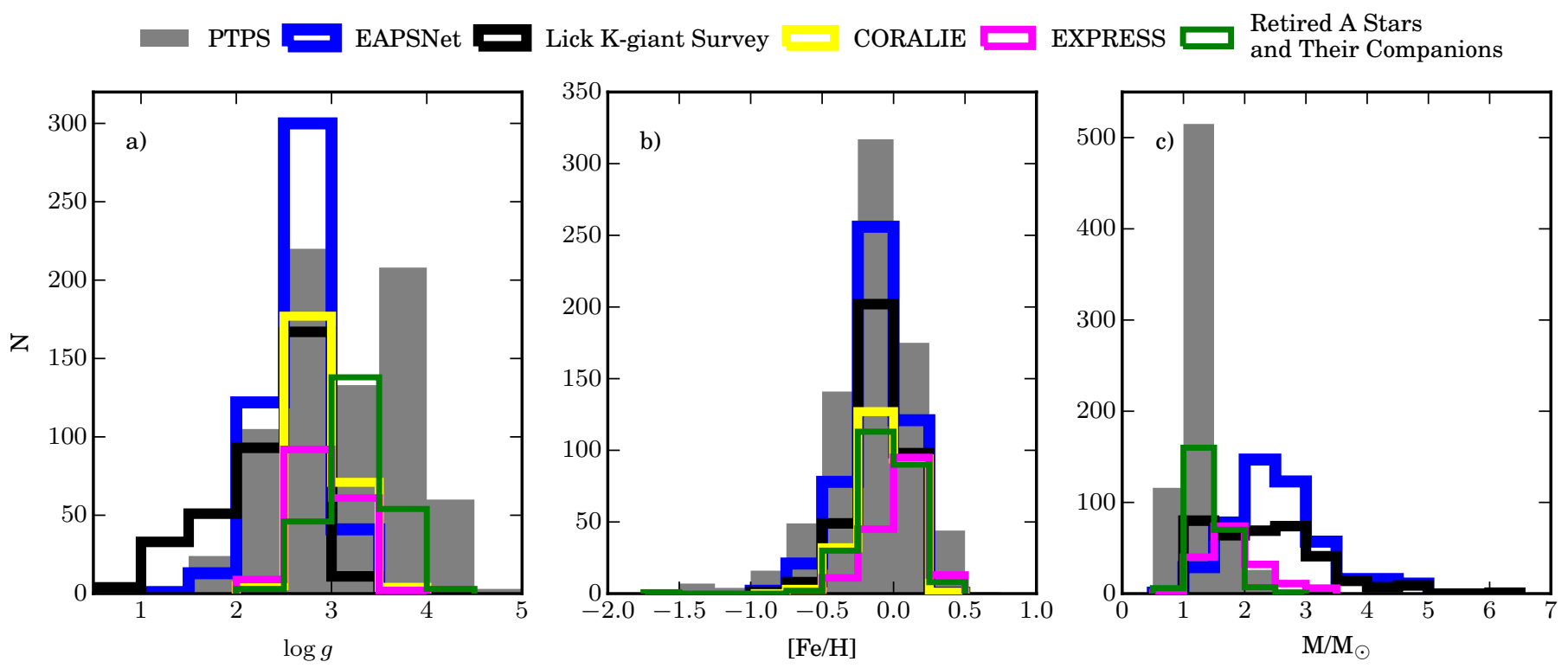

Fig. 13. Histograms for $\log g,[\mathrm{Fe} / \mathrm{H}]$, and $M / M_{\odot}$ for planet search projects around evolved stars.
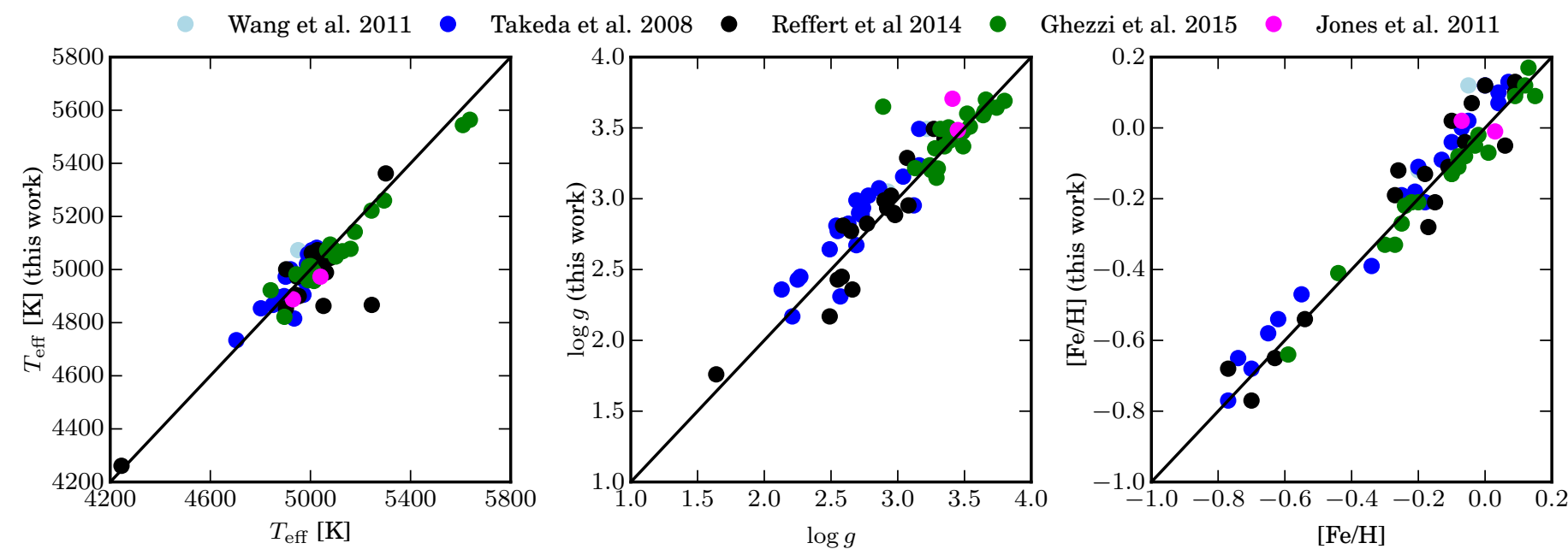

Fig. 14. Comparison of stellar atmospheres for 62 PTPS stars that are also included in other planet searches.

The reason for the fast mass increase of the companions of evolved stars remains elusive. We have shown in Sect. 6 that in some planet searches around evolved stars the stellar masses may be overestimated by up to $43 \%$.

In Table 4 we compile the stellar masses for evolved planetary system hosts from the list of Reffert et al. (2014) and all hosts of planetary systems detected within PTPS. The stellar masses estimated here are higher for the Retired Stars and Their Companions and the Lick K-giants Survey. The ratio of original to stellar masses derived here is $0.91 \pm 0.09$ and $0.93 \pm 0.15$. In all other surveys the stellar masses are overestimated relative to our determinations, and the respective mass ratios are $1.13 \pm 0.22$ for EXPRESS, $1.42 \pm 0.31$ for EAPSNet, and $1.14 \pm 0.45$ for PTPS. On average, however, the stellar masses for evolved planetary systems included in the compilation of Reffert et al. (2014) are only very slightly overestimated, with the ratio of the original to the average mass presented here being $1.12 \pm 0.35$.

Figure 16 shows that companions masses, recalculated with our estimates of hosts masses for all systems from the compilation of Reffert et al. (2014), generally agree with the originally published values, with very few exceptions. This is expected because the $12 \%$ overestimate of stellar host masses contributes as $\sim m^{2 / 3}$ to companions masses. Therefore the apparent overestimate of stellar host masses in several planet search projects does not result in an overestimate of companion masses.

In Table 5 we collected from exoplanet.eu the basic data for planetary systems around evolved stars $(\log g<3)$ that were discovered with the RV technique according to the same selection criteria as in Niedzielski et al. (2015b). For all these systems we calculated new stellar masses of the stellar host, including our own detections, based on available atmospheric parameters. We also scaled masses of their companions assuming the new host masses. We found that for individual system both an increase and a decrease of the companion masses may occur. We also calculated the median, mean, and standard deviations within $\log g=2.5 \pm 0.5$ (giants, 44 stars) and $\log g=1.5 \pm 0.5$ (bright giants, 16 stars). For giants and bright giants, the mean, median, and standard deviations of companion masses are 5.46, $3.33,0.84$ and $7.89,6.38$ and 1.12 . We found that the low-mass companion masses around giants show lower mean masses than was found by Niedzielski et al. (2015b), but both values agree within $1 \sigma$. For bright giants the mean companion mass remains 

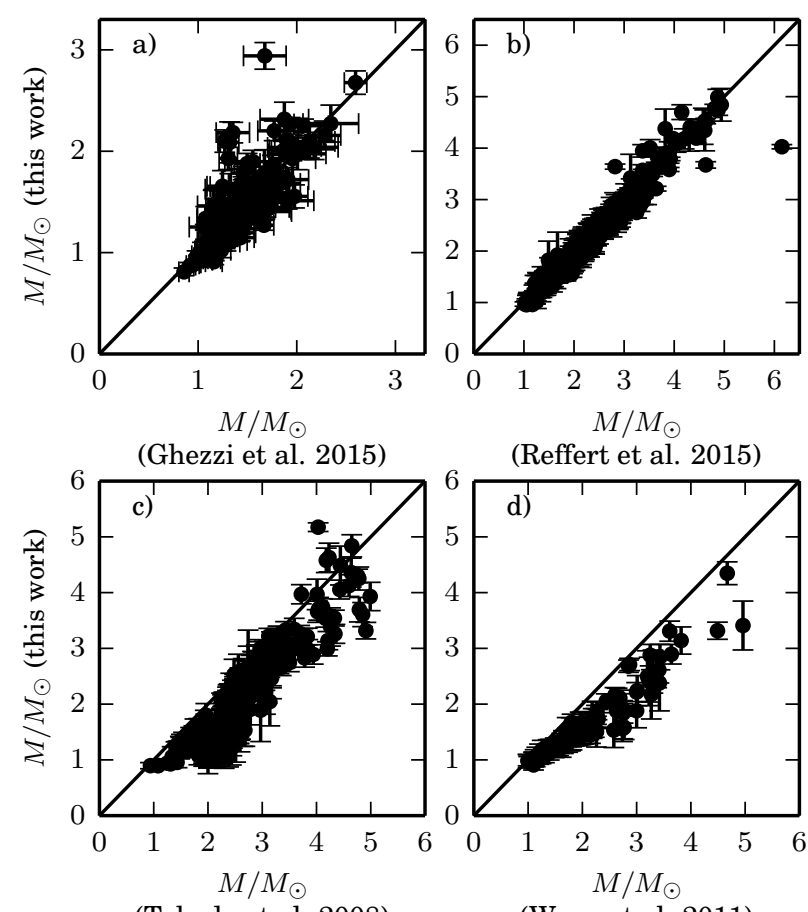

(Reffert et al. 2015)
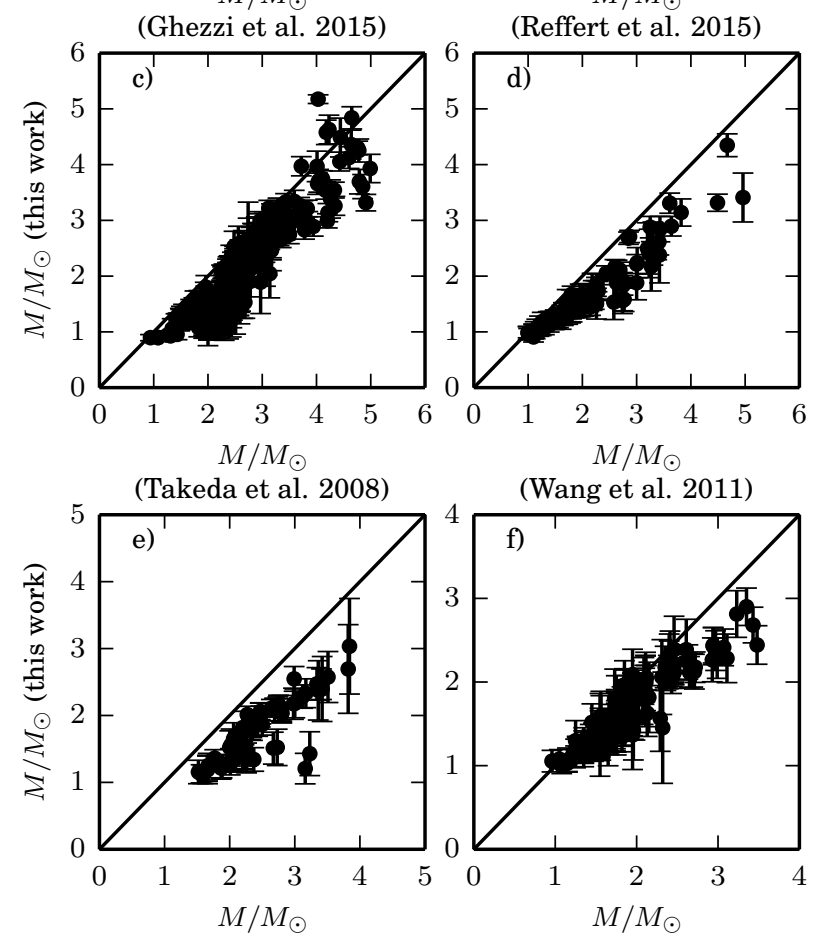

(Wang et al. 2011)

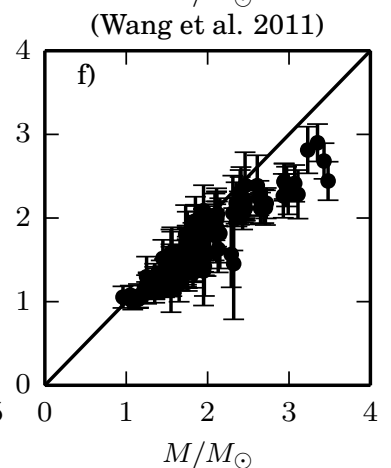

(Jones et al. 2011)

Fig. 15. Comparison of stellar masses presented by different authors with masses calculated by us using published stellar atmospheric parameters. Stellar masses presented by Reffert et al. (2014) in the Lick K-giant Survey and by Ghezzi \& Johnson (2015) in the Retired A Stars and Their Companions projects agree well with our estimates, while stellar masses presented by other authors disagree with our estimates.

essentially the same. Again, the evident overestimate of stellar hosts masses in several planet search projects does not seem to be the reason of the apparent companions mass increase for evolved stars.

We therefore conclude that even with stellar masses corrected for possible overestimates, existing data show virtually the same increase in companion mass for evolved stars as presented in Niedzielski et al. (2015b). We are therefore confident that the apparent increase in companion mass for evolved stars is either real or caused by other factors than overestimated host masses.

Obviously, sample definitions in planet search projects devoted to evolved stars, especially the abundance of relatively massive targets, may play a role. There may be more simple reasons, however. First of all, they may be of instrumental nature, such as limited actual precision of RV measurements, because not all surveys delivered companions in the $1 M_{\mathrm{J}}$ mass range. The stellar jitter, unresolved in time $p$-mode oscillations (Kjeldsen \& Bedding 1995), which in evolved stars are expected

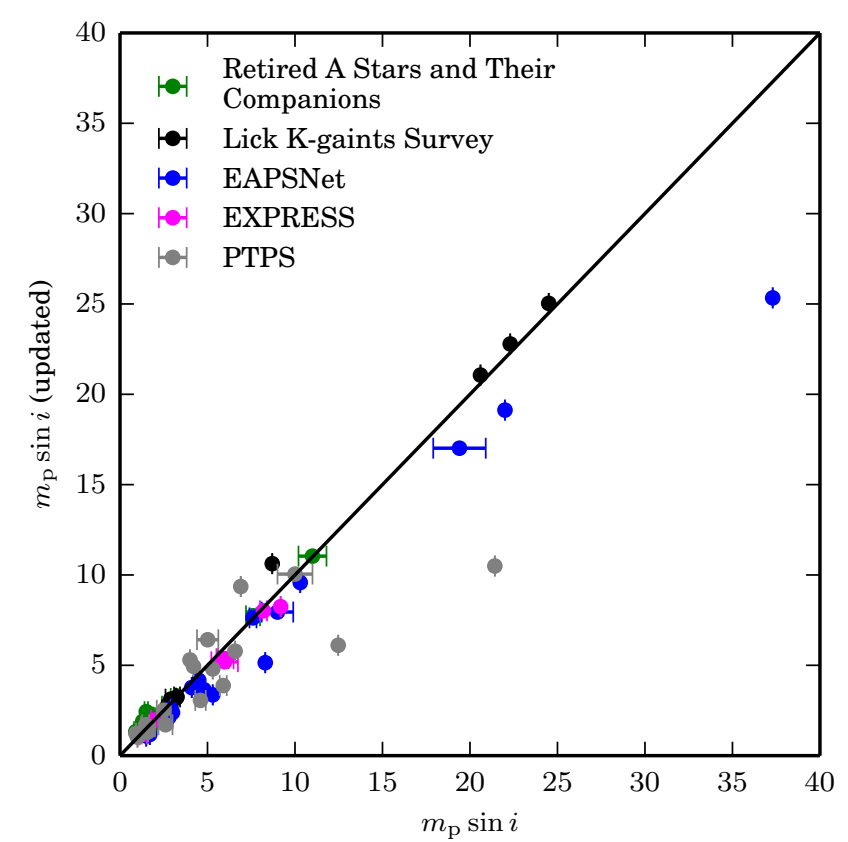

Fig. 16. Comparison of companion masses originally published and calculated with our Bayesian approach. Systems listed in the compilation of Reffert et al. (2014) are presented. Most companions show masses very similar to the originally published values.

to reach $\sim 100 \mathrm{~m} \mathrm{~s}^{-1}$, might be another factor limiting the actual, effective RV precision when applied to luminous stars. Although it has been demonstrated by various authors that with systematic observations of such stars companions of $\leq 1 M_{\mathrm{J}}$ can unequivocally be detected (Gettel et al. 2012a,b; Nowak et al. 2013), it is tempting to try to study these two factors in more detail.

In a search for a simple explanation of the apparent mass increase in low-mass companions for evolved stars we assumed that the effective RV precision of a planet search is

$\sigma_{\mathrm{e}}=\sqrt{\sigma_{\mathrm{RV}}^{2}+\text { jitter }^{2}}$,

where $\sigma_{\mathrm{RV}}$ is the instrumental $\mathrm{RV}$ precision and jitter is the amplitude of $p$-mode oscillations for given stellar mass and luminosity according to Kjeldsen \& Bedding (1995). We also assumed that within a planet search a low-mass companion can be detected if the RV semiamplitude $K \geq K_{\mathrm{c}}=3 \times \sigma_{\mathrm{e}}$. In Fig. 17 we present the resulting $K_{\mathrm{c}}$ at various orbital separations for $\sigma_{\mathrm{RV}}=10 \mathrm{~m} \mathrm{~s}^{-1}$, average stellar host masses for dwarfs, subgiants, giants, and bright giants from Niedzielski et al. (2015b); and assuming (arbitrary) luminosities of $L / L_{\odot}=1,25,200$, and 500 , respectively.

There are two immediate conclusions obvious from Fig. 17: (i) the minimum detectable companion mass increases with orbital separation and for evolved more active stars; and (ii) for evolved stars the detection of nearby low-mass companions $\left(m_{\mathrm{p}} \leq 2 M_{\mathrm{J}}\right.$ within $\left.1 \mathrm{AU}\right)$ is difficult, if possible at all.

Figure 17 shows that the planetary mass distributions for dwarfs and subgiants are very similar because the detectabillity of companions to those stars is mainly limited by the instrumental RV precision. Moreover, the increase of the minimum detectable companions masses for evolved stars comes naturally from the increased level of additional stellar noise. Assuming further a uniform distribution of companions orbit with $5 \mathrm{AU}$ for simplicity, we obtained average minimum masses (in $M_{\mathrm{J}}$ ) of detectable companions to dwarfs, subgiants, giants, and bright 


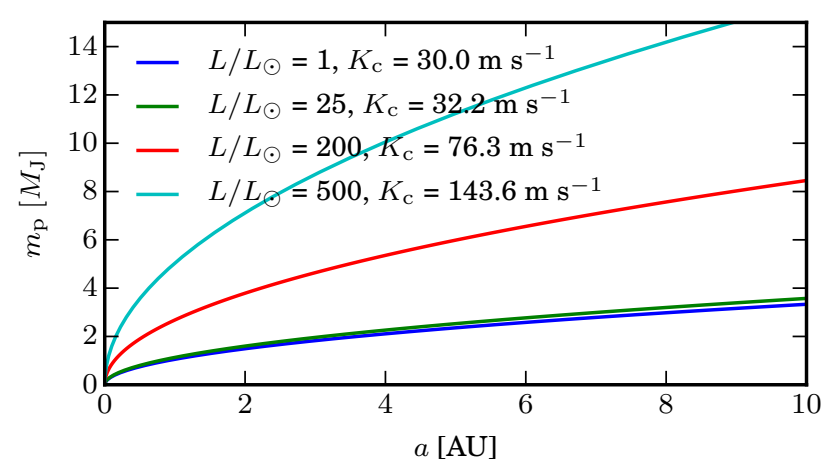

Fig. 17. Minimum detectable companion masses vs. semimajor axis for stars at various evolutionary stages. See text for explanation.

giants of $1.2,1.3,3.0$, and 5.6, respectively, which qualitatively agrees with observations.

Conclusion (ii) above suggests that the apparent lack of planets within $~ 0.5 \mathrm{AU}$ around evolved stars (Johnson et al. 2007; Sato et al. 2008) may not be a physical phenomenon, but only a reflection of the limited effective RV precision, if for some reason companions more massive than $\sim 2 M_{\mathrm{J}}$ are not so abundant in close-in orbits around evolved stars (an assumption very well supported by observations). This finding seems also to be supported by the recent discovery of Kepler $91 \mathrm{~b}$, a $0.66 \pm 0.06 M_{\mathrm{J}}$ planet in only $a / R_{\star}=2.253 \pm 0.046\left(R_{\star}=6.30 \pm 0.16 R_{\odot}\right)$ orbit around a $1.3 \pm 0.1 M_{\odot}, \log g=2.953 \pm 0.07$ giant (Lillo-Box et al. 2014; Barclay et al. 2015; Sato et al. 2015).

If we assume that low-mass companions around giants and bright giants are present outside $0.5 \mathrm{AU}$ only, the average minimum companion masses to those stars grow to 3.9 and 7.4 , respectively, which makes our conclusion even stronger.

Based on instrumental precision and stellar activity alone, we can at least qualitatively explain the observed apparent increase of companion masses for evolved stars and question the reality of the apparent lack of low-mass companions to evolved stars within 0.5 AU.

\section{Conlusions}

We have presented atmospheric parameters, luminosities, masses, stellar ages, and radii for 402 stars from the PTPS. We also presented estimated atmospheric parameters for another 53 stars that originally were included in the PTPS, but were rejected as unsuitable for a planet search. For 272 stars the results are first determinations. We presented and discussed in more detail the complete sample of 744 stars beyond the MS that form the PTPS sample of evolved stars.

To explain the apparent increase in planetary mass of the companions to evolved stars, we compiled atmospheric parameters for 1255 stars from another five planet searches and estimated stellar masses in a uniform way. Our results agree very well with the Lick K-giant Survey of Reffert et al. (2014) and the Retired A stars and their Companions survey (Ghezzi \& Johnson 2015), but we also found that stellar masses presented in two other projects appear to be seriously overestimated.

We do not claim that our estimates of stellar masses are better, more precise, or more reliable. The only point we make is that the problem of stellar masses of evolved planetary system hosts indeed exists. We stress that the stellar masses estimated by us are most likely upper limits, which challenges the stellar mass estimates of the several other samples; it may be worthwhile to reanalyze them.
With uniformly determined stellar masses we checked whether the apparent planetary mass increase for evolved stars can be real, and we found that it is not the result of the overestimated stellar mass reported by some authors.

We demonstrated that the apparent increase of the companion masses of evolved stars and the lack of planetary mass companions to evolved stars within $0.5 \mathrm{AU}$ may both originate from limited RV precision and additional noise introduced by stellar $p$-mode oscillations.

Acknowledgements. We thank Yoichi Takeda for making his codes available to us and Luan Ghezzi for sharing his preliminary results prior to publication. We thank the HET resident astronomers and telescope operators for their continuous support. We thank the anonymous referee for comments that let us improve the paper substantially. Mi.A., A.N., B.S.-D., M.A., and G.N. were supported by the Polish National Science Centre grant no. UMO-2012/07/B/ST9/04415. M.A. acknowledges the Mobility+III fellowship from the Polish Ministry of Science and Higher Education. The Hobby Eberly Telescope (HET) is a joint project of the University of Texas at Austin, the Pennsylvania State University, Stanford University, Ludwig-Maximilians-Universität München, and GeorgAugust-Universität Göttingen. The HET is named in honor of its principal benefactors, William P. Hobby and Robert E. Eberly. The Center for Exoplanets and Habitable Worlds is supported by the Pennsylvania State University, the Eberly College of Science, and the Pennsylvania Space Grant Consortium. This research has made extensive use of the SIMBAD database, operated at CDS (Strasbourg, France) and NASA's Astrophysics Data System Bibliographic Services.

\section{References}

Adamczyk, M., Deka-Szymankiewicz, B., \& Niedzielski, A. 2015, A\&A, accepted

Adamów, M., \& Niedzielski, A. 2010, in EAS PS 42, eds. K. Gożdziewski, A. Niedzielski, \& J. Schneider, 91

Adamów, M., Niedzielski, A., Villaver, E., Nowak, G., \& Wolszczan, A. 2012, ApJ, 754, L15

Adamów, M., Niedzielski, A., Villaver, E., Wolszczan, A., \& Nowak, G. 2014, A\&A, 569, A55

Adamów, M., M., Niedzielski, A., Villaver, E., et al. 2015, A\&A, 581, A94

Almenara, J. M., Damiani, C., Bouchy, F., et al. 2015, A\&A, 575, A71

Alonso, A., Arribas, S., \& Martínez-Roger, C. 1999, A\&AS, 140, 261

Alves, S., Benamati, L., Santos, N. C., et al. 2015, MNRAS, 448, 2749

Barclay, T., Endl, M., Huber, D., et al. 2015, ApJ, 800, 46

Bertelli, G., Girardi, L., Marigo, P., \& Nasi, E. 2008, A\&A, 484, 815

Bertelli, G., Nasi, E., Girardi, L., \& Marigo, P. 2009, A\&A, 508, 355

Bieryla, A., Collins, K., Beatty, T. G., et al. 2015, AJ, 150, 12

Bilir, S., Karaali, S., Güver, T., Karataş, Y., \& Ak, S. G. 2006, Astron. Nachr., 327, 72

Borucki, W. J., Koch, D. G., Basri, G., et al. 2011, ApJ, 728, 117

Bowler, B. P., Johnson, J. A., Marcy, G. W., et al. 2010, ApJ, 709, 396

Bressan, A., Marigo, P., Girardi, L., et al. 2012, MNRAS, 427, 127

Butler, R. P., Wright, J. T., Marcy, G. W., et al. 2006, ApJ, 646, 505

Caffau, E., Ludwig, H.-G., Steffen, M., Freytag, B., \& Bonifacio, P. 2011, Sol. Phys., 268, 255

Ciceri, S., Lillo-Box, J., Southworth, J., et al. 2015, A\&A, 573, L5

Cochran, W. D., \& Hatzes, A. P. 1993, in Planets Around Pulsars, eds.

J. A. Phillips, S. E. Thorsett, \& S. R. Kulkarni, ASP Conf. Ser., 36, 267

da Silva, L., Girardi, L., Pasquini, L., et al. 2006, A\&A, 458, 609

Duncan, M. J., \& Lissauer, J. J. 1998, Icarus, 134, 303

Frink, S., Mitchell, D. S., Quirrenbach, A., et al. 2002, ApJ, 576, 478

Gelino, C. R., Shao, M., Tanner, A. M., \& Niedzielski, A. 2005, in Protostars and Planets V, $8602 \mathrm{~s}$

Gettel, S., Wolszczan, A., Niedzielski, A., et al. 2012a, ApJ, 756, 53

Gettel, S., Wolszczan, A., Niedzielski, A., et al. 2012b, ApJ, 745, 28

Ghezzi, L., \& Johnson, J. 2015, IAU General Assembly, 22, 58066

Gilmore, G. F., Perryman, M. A., Lindegren, L., et al. 1998, in Astronomical Interferometry, ed. R. D. Reasenberg, SPIE Conf. Ser., 3350, 541

Hartman, J. D., Bakos, G. Á., Béky, B., et al. 2012, AJ, 144, 139

Hatzes, A. P., \& Cochran, W. D. 1993, ApJ, 413, 339

Hatzes, A. P., Guenther, E. W., Endl, M., et al. 2005, A\&A, 437, 743

Holweger, H., Bard, A., Kock, M., \& Kock, A. 1991, A\&A, 249, 545

Ibukiyama, A., \& Arimoto, N. 2002, A\&A, 394, 927

Johnson, D. R. H., \& Soderblom, D. R. 1987, AJ, 93, 864

Johnson, J. A., Fischer, D. A., Marcy, G. W., et al. 2007, ApJ, 665, 785

Jones, M. I., Jenkins, J. S., Rojo, P., \& Melo, C. H. F. 2011, A\&A, 536, A71

Jones, M. I., Jenkins, J. S., Bluhm, P., Rojo, P., \& Melo, C. H. F. 2014, A\&A, 566, A113 
Jørgensen, B. R., \& Lindegren, L. 2005, A\&A, 436, 127

Kjeldsen, H., \& Bedding, T. R. 1995, A\&A, 293, 87

Kurucz, R. 1993, ATLAS9 Stellar Atmosphere Programs and $2 \mathrm{~km} \mathrm{~s}^{-1}$ grid. Kurucz CD-ROM No. 13 (Cambridge, MA: Smithsonian Astrophysical Observatory)

Lee, B.-C., Mkrtichian, D. E., Han, I., Kim, K.-M., \& Park, M.-G. 2011, A\&A, $529, \mathrm{~A} 134$

Lillo-Box, J., Barrado, D., Moya, A., et al. 2014, A\&A, 562, A109

Liu, Y., Sato, B., Takeda, Y., Ando, H., \& Zhao, G. 2010, PASJ, 62, 1071

Lloyd, J. P. 2011, ApJ, 739, L49

Lloyd, J. P. 2013, ApJ, 774, L2

Lovis, C., \& Mayor, M. 2007, A\&A, 472, 657

Marcy, G. W., \& Butler, R. P. 1996, ApJ, 464, L147

Martin, P. 1977, A\&A, 61, 591

Mayor, M., \& Queloz, D. 1995, Nature, 378, 355

Miglio, A., Brogaard, K., Stello, D., et al. 2012, MNRAS, 419, 2077

Mitchell, D. S., Reffert, S., Trifonov, T., Quirrenbach, A., \& Fischer, D. A. 2013, A\&A, 555, A87

Mortier, A., Santos, N. C., Sousa, S. G., et al. 2013, A\&A, 557, A70

Niedzielski, A., \& Wolszczan, A. 2008, in IAU Symp. 249, eds. Y.-S. Sun, S. Ferraz-Mello, \& J.-L. Zhou, 43

Niedzielski, A., Konacki, M., Wolszczan, A., et al. 2007, ApJ, 669, 1354

Niedzielski, A., Goździewski, K., Wolszczan, A., et al. 2009a, ApJ, 693, 276

Niedzielski, A., Nowak, G., Adamów, M., \& Wolszczan, A. 2009b, ApJ, 707, 768

Niedzielski, A., Wolszczan, A., Adamów, M., et al. 2013, Mem. Soc. Astron. It., 84,1035

Niedzielski, A., Villaver, E., Wolszczan, A., et al. 2015a, A\&A, 573, A36

Niedzielski, A., Wolszczan, A., Nowak, G., et al. 2015b, ApJ, 803, 1

Nowak, G. 2012, Ph.D. Thesis, Nicolaus Copernicus Univ., Toruń, Poland

Nowak, G., Niedzielski, A., Wolszczan, A., Adamów, M., \& Maciejewski, G. 2013, ApJ, 770, 53

Ortiz, M., Gandolfi, D., Reffert, S., et al. 2015, A\&A, 573, L6

Perryman, M. A. C., de Boer, K. S., Gilmore, G., et al. 2001, A\&A, 369, 339

Quinn, S. N., White, T. R., Latham, D. W., et al. 2015, ApJ, 803, 49

Ramírez, I., \& Allende Prieto, C. 2011, ApJ, 743, 135

Ramírez, I., \& Meléndez, J. 2005, ApJ, 626, 446

Ramsey, L. W., Adams, M. T., Barnes, T. G., et al. 1998, in SPIE Conf. Ser. 3352, ed. L. M. Stepp, 34

Rauer, H., Catala, C., Aerts, C., et al. 2014, Exp. Astron. 38, 249
Reffert, S., Bergmann, C., Quirrenbach, A., Trifonov, T., \& Kuenstler, A. 2014, VizieR Online Data Catalog: J/A+A/574/A116

Ricker, G. R., Winn, J. N., Vanderspek, R., et al. 2014, in SPIE Conf. Ser. 9143, 20

Rieke, G. H., \& Lebofsky, M. J. 1985, ApJ, 288, 618

Sato, B., Ando, H., Kambe, E., et al. 2003, ApJ, 597, L157

Sato, B., Izumiura, H., Toyota, E., et al. 2007, ApJ, 661, 527

Sato, B., Izumiura, H., Toyota, E., et al. 2008, PASJ, 60, 539

Sato, B., Omiya, M., Liu, Y., et al. 2010, PASJ, 62, 1063

Sato, B., Omiya, M., Harakawa, H., et al. 2012, PASJ, 64, 135

Sato, B., Omiya, M., Harakawa, H., et al. 2013, PASJ, 65, 85

Sato, B., Hirano, T., Omiya, M., et al. 2015, ApJ, 802, 57

Schlaufman, K. C., \& Winn, J. N. 2013, ApJ, 772, 143

Schwamb, M. E., Orosz, J. A., Carter, J. A., et al. 2013, ApJ, 768, 127

Setiawan, J., Hatzes, A. P., von der Lühe, O., et al. 2003a, A\&A, 398, L19

Setiawan, J., Pasquini, L., da Silva, L., von der Lühe, O., \& Hatzes, A. 2003b, A\&A, 397, 1151

Shetrone, M., Cornell, M. E., Fowler, J. R., et al. 2007, PASP, 119, 556

Smalley, B., Anderson, D. R., Collier-Cameron, A., et al. 2012, A\&A, 547, A61

Soubiran, C., Katz, D., \& Cayrel, R. 1998, A\&AS, 133, 221

Soubiran, C., Le Campion, J.-F., Cayrel de Strobel, G., \& Caillo, A. 2010, A\&A, 515, A111

Sousa, S. G., Santos, N. C., Israelian, G., Mayor, M., \& Monteiro, M. J. P. F. G. 2007, A\&A, 469, 783

Sousa, S. G., Santos, N. C., Mortier, A., et al. 2015, A\&A, 576, A94

Stetson, P. B., \& Pancino, E. 2008, PASP, 120, 1332

Stumpff, P. 1980, A\&AS, 41, 1

Takeda, Y., Ohkubo, M., \& Sadakane, K. 2002, PASJ, 54, 451

Takeda, Y., Ohkubo, M., Sato, B., Kambe, E., \& Sadakane, K. 2005, PASJ, 57, 27

Takeda, Y., Sato, B., \& Murata, D. 2008, PASJ, 60, 781

Tsantaki, M., Sousa, S. G., Adibekyan, V. Z., et al. 2013, A\&A, 555, A150

Tull, R. G. 1998, in SPIE Conf. Ser. 3355, ed. S. D’Odorico, 387

Valenti, J. A., \& Piskunov, N. 1996, A\&AS, 118, 595

van Leeuwen, F. 2007, A\&A, 474, 653

Wang, L., Liu, Y., Zhao, G., \& Sato, B. 2011, PASJ, 63, 1035

Wolszczan, A., \& Frail, D. A. 1992, Nature, 355, 145

Zieliński, P., Niedzielski, A., Wolszczan, A., Adamów, M., \& Nowak, G. 2012, A\&A, 547, A91 